\title{
Functorial seminorms on singular homology and (in)flexible manifolds
}

\author{
DIARMUID CROWLEY \\ ClarA LÖH
}

\begin{abstract}
A functorial seminorm on singular homology is a collection of seminorms on the singular homology groups of spaces such that continuous maps between spaces induce norm-decreasing maps in homology. Functorial seminorms can be used to give constraints on the possible mapping degrees of maps between oriented manifolds. In this paper, we use information about the degrees of maps between manifolds to construct new functorial seminorms with interesting properties. In particular, we answer a question of Gromov by providing a functorial seminorm that takes finite positive values on homology classes of certain simply connected spaces. Our construction relies on the existence of simply connected manifolds that are inflexible in the sense that all their self-maps have degree $-1,0$ or 1 . The existence of such manifolds was first established by Arkowitz and Lupton; we extend their methods to produce a wide variety of such manifolds.
\end{abstract}

$57 \mathrm{~N} 65,55 \mathrm{~N} 10 ; 55 \mathrm{~N} 35,55 \mathrm{P} 62$

\section{Introduction}

Enriching algebraic invariants with metric data is a common theme in many branches of mathematics. Gromov introduced the concept of functorial seminorms on singular homology [11, Section 5.34], which are an example of this paradigm in topology.

A functorial seminorm on singular homology consists of the addition of a seminormed structure to the singular homology groups with $\mathbb{R}$-coefficients in such a way that continuous maps induce linear maps on homology of norm at most 1 (Definition 2.1). An interesting aspect is that suitable functorial seminorms give a systematic way to deduce degree theorems for maps between manifolds (Remark 2.6). Conversely, in the present paper, we translate knowledge about degrees of maps between manifolds to construct new functorial seminorms.

A central example of a functorial seminorm on singular homology, studied by Gromov [10], is the $\ell^{1}$-seminorm given by taking the infimum of the $\ell^{1}$-norms of all 
cycles representing a given homology class (Example 2.2). The $\ell^{1}$-seminorm gives rise to lower bounds for the minimal volume and hence leads to interesting applications in Riemannian geometry [10]. On the other hand, using bounded cohomology, Gromov showed that the $\ell^{1}$-seminorm vanishes on classes of nonzero degree of simply connected spaces [10], and later raised the question whether every functorial seminorm on singular homology in nonzero degree is trivial on all simply connected spaces [11, Remark(b) in 5.35]. More precisely, we formulate this problem as follows:

Question 1.1 Let $d \in \mathbb{N}_{>0}$.

(1) Does every (possibly infinite) functorial seminorm on singular homology in degree $d$ take only the values 0 and $\infty$ on homology classes of simply connected spaces?

(2) Does every finite functorial seminorm on singular homology in degree $d$ vanish on homology classes of simply connected spaces?

In this paper, we answer the first part of this question in the negative (Corollary 7.4):

Theorem 1.2 There are functorial seminorms on singular homology that are positive and finite on certain homology classes of simply connected spaces.

More concretely, we give examples of such functorial seminorms in all degrees in the set $\{64\} \cup\left\{d \cdot k \mid k \in \mathbb{N}_{>0}, d \in\{108,208,228\}\right\}$ (Corollary II.7).

On the other hand, we give a positive answer to Question 1.1(2) in low dimensions (Section 7.2):

Theorem 1.3 All finite functorial seminorms on singular homology in degrees $1, \ldots, 6$ vanish on all homology classes of simply connected spaces.

The key to proving Theorems 1.2 and 1.3 is to gain an understanding of the class of simply connected inflexible manifolds.

Definition 1.4 (Inflexible manifolds) If $M$ and $N$ are oriented closed connected manifolds of the same dimension, then we write

$$
\operatorname{deg}(N, M):=\{\operatorname{deg} f \mid f: N \rightarrow M \text { continuous }\}
$$

for the set of all possible mapping degrees for maps from $N$ to $M$. An oriented closed connected manifold $M$ is inflexible if $\operatorname{deg}(M, M) \subset\{-1,0,1\}$. 
The proof of Theorem 1.2 consists of two main steps.

- Generating functorial seminorms via manifolds Using the fact that singular homology classes can (up to a scalar multiple) be represented by fundamental classes of oriented closed connected manifolds (Section 3), we show how functorial seminorms on fundamental classes of manifolds of a given dimension can be extended to functorial seminorms on singular homology (Theorem 4.2).

- Inflexible manifolds With the help of simply connected inflexible manifolds, we construct a functorial seminorm on fundamental classes of manifolds that is positive and finite on the given simply connected inflexible manifold (Corollary 7.4).

Simply connected inflexible manifolds can be constructed by means of rational homotopy theory and surgery theory. The first examples of such manifolds were given by Arkowitz and Lupton [2, Examples 5.1 and 5.2]; these examples have dimension 208 and 228 respectively. Using and extending the methods of Arkowitz and Lupton, we give more examples of simply connected inflexible manifolds. For instance, we have examples in dimension 64 (the smallest dimension known before being 208) and 108 . Starting from these basic examples, we can construct many more simply connected inflexible manifolds.

- In general, it is not clear that connected sums and products of inflexible manifolds are inflexible; however, in certain cases this is true (Sections II.1 and II.2). This provides in infinitely many dimensions infinitely many rational homotopy types of oriented closed simply connected inflexible manifolds (Corollary II.7).

- In addition, using scaling of the fundamental class with respect to a rationalisation, we obtain infinitely many homotopy types of oriented closed simply connected inflexible manifolds within the same rational homotopy type (Proposition II.8).

- Moreover, we can show that for manifolds being simply connected and inflexible is generic in the sense that in infinitely many dimensions every rational bordism class is represented by a simply connected inflexible manifold (Proposition II.12).

- Also, there are simply connected inflexible smooth manifolds satisfying certain tangential structure constraints such as being stably parallelisable or nonspinable (Section II.3).

However, from our construction it is not clear whether the examples from Theorem 1.2 are finite functorial seminorms; so Gromov's question remains open for finite functorial seminorms in degree 7 and higher. More precisely, we prove the following proposition (Proposition 7.6) where an oriented closed connected $n$-manifold $M$ is called strongly inflexible if for any oriented closed connected $n$-manifold $N$ the set $\operatorname{deg}(N, M)$ is finite (Definition 6.14): 
Proposition 7.6 For $d \in \mathbb{N}_{\geq 4}$ the following statements are equivalent.

(1) There is a finite functorial seminorm $|\cdot|$ on $H_{d}(\cdot ; \mathbb{R})$ such that for some homology class $\alpha \in H_{d}(X ; \mathbb{R})$ of some simply connected space $X$ we have $|\alpha| \neq 0$.

(2) There exists an oriented closed simply connected $d$-manifold that is strongly inflexible.

No example of a simply connected strongly inflexible manifold seems to be known to date: if such a manifold exists, it has dimension at least 7 .

Remark 1.5 Since this paper was posted Costoya and Viruel [6] and also Amann [1] have further extended the list of examples and constructions of simply connected inflexible manifolds. Amann [1] has also given new examples of simply connected flexible manifolds.

Organisation of this paper We start by giving an introduction to functorial seminorms (Section 2). In Section 3 we recall Thom's result on representation of homology classes by fundamental classes of manifolds, which is the key ingredient for generating functorial seminorms via functorial seminorms for manifolds (Section 4). We discuss the relationship between functorial seminorms on the singular chain complex and functorial seminorms on singular homology in Section 5. In Section 7 we prove Theorems 1.2 and 1.3. The proof of Theorem 1.2 is based on the construction of simply connected inflexible manifolds; we carefully review and extend the construction of Arkowitz and Lupton of simply connected inflexible manifolds in Section 6, the technical aspects being deferred to Appendix I. Finally, Appendix II contains the study of inheritance properties of being inflexible and evidence for the genericity of inflexibility in the class of simply connected manifolds.

Acknowledgements We are indebted to Donald Stanley who drew our attention to the examples of Arkowitz and Lupton. Moreover, we would like to thank Thomas Schick for interesting discussions. We are grateful to Jonathan Bowden for pointing out a mistake in a previous version. Part of this work was supported by the HIM trimester program Rigidity and by the SFB 878 Groups, Geometry and Actions.

\section{Functorial seminorms}

Functorial seminorms assign a notion of "size" to singular homology classes in a functorial way (Definition 2.1). 
In this paper, we use the following convention: a seminorm on an $\mathbb{R}$-vector space $V$ is a function $|\cdot|: V \rightarrow[0, \infty]$ satisfying the following properties.

- We have $|0|=0$.

- For all $x \in V$ and all $a \in \mathbb{R} \backslash\{0\}$, we have $|a \cdot x|=|a| \cdot|x|$, where $|a| \cdot \infty:=\infty$.

- For all $x, y \in V$ the triangle inequality $|x+y| \leq|x|+|y|$ holds.

A seminorm is called finite if it does not take the value $\infty$.

Definition 2.1 (Functorial seminorms [11, Section 5.34]) Let $d \in \mathbb{N}$. A functorial seminorm (on singular homology) in degree $d$ consists of a choice of a seminorm $|\cdot|$ on $H_{d}(X ; \mathbb{R})$ for any topological space $X$ such that the following "functoriality" holds: for all continuous maps $f: X \rightarrow Y$ between topological spaces and all $\alpha \in H_{d}(X ; \mathbb{R})$ we have

$$
\left|H_{d}(f ; \mathbb{R})(\alpha)\right| \leq|\alpha| .
$$

Such a functorial seminorm is called finite if all the seminorms involved are finite seminorms.

Example 2.2 $\left(\ell^{1}\right.$-seminorm) For a topological space $X$ let $|\cdot|_{1}$ denote the $\ell^{1}-$ norm on the singular chain complex $C_{*}(X ; \mathbb{R})$ with respect to the (unordered) basis given by all singular simplices: if $c=\sum_{j=1}^{k} a_{j} \cdot \sigma_{j} \in C_{*}(X ; \mathbb{R})$ is in reduced form, then we define

$$
|c|_{1}:=\sum_{j=1}^{k}\left|a_{j}\right| .
$$

This norm induces a finite seminorm $\|\cdot\|_{1}$, the so-called $\ell^{1}$-seminorm, on singular homology as follows: for all $\alpha \in H_{*}(X ; \mathbb{R})$ we set

$$
\|\alpha\|_{1}:=\inf \left\{|c|_{1} \mid c \in C_{*}(X ; \mathbb{R}) \text { is a cycle representing } \alpha\right\} .
$$

Looking at the definition of the homomorphisms induced by continuous maps in singular homology, it is immediate that $\|\cdot\|_{1}$ is a functorial seminorm on singular homology.

An interesting topological invariant derived from the $\ell^{1}$-seminorm in singular homology is the simplicial volume, introduced by Gromov [11]: if $M$ is an oriented closed connected manifold, then

$$
\|M\|:=\left\|[M]_{\mathbb{R}}\right\|_{1} \in \mathbb{R}_{\geq 0}
$$

is the simplicial volume of $M$, where $[M]_{\mathbb{R}} \in H_{\operatorname{dim} M}(M ; \mathbb{R})$ denotes the $\mathbb{R}$-fundamental class of $M$. For example, using self-maps of nontrivial degree, one sees that 
the simplicial volume of spheres (of nonzero dimension) is zero. On the other hand, for example, the simplicial volume of oriented closed connected hyperbolic manifolds is nonzero ([10, Section 0.3] and Thurston [24, Theorem 6.2]), leading to interesting applications in Riemannian geometry [10].

The $\ell^{1}$-seminorm on singular homology can also be expressed in terms of bounded cohomology ([10, page 17] and Benedetti and Petronio [5, Proposition F.2.2]). Using bounded cohomology, Gromov discovered that the $\ell^{1}$-seminorm of simply connected spaces is trivial ([10, Section 3.1] and Ivanov [12, Theorem 2.4]) and, more generally, that continuous maps that induce an isomorphism on the level of fundamental groups induce norm-preserving maps on the level of singular homology ([10, Section 3.1] and Ivanov [12, Theorem 4.3]).

It is tempting to analogously consider $\ell^{p}$-norms with $p>1$; however, it can be shown that the corresponding definition then leads to the zero seminorm on homology in positive degrees (this follows from an argument similar to (Non)example 5.1)

Example 2.3 (Domination by products of surfaces) For $d \in \mathbb{N}$, we define the functorial seminorm $|\cdot|_{S}$ in degree $2 d$ as follows [11, Section 5.34]. Let $X$ be a topological space, and let $\alpha \in H_{2 d}(X ; \mathbb{R})$. Then

$|\alpha|_{S}:=\inf \left\{\begin{array}{c|c}\sum_{j=1}^{k}\left|a_{j}\right| \cdot\left|\chi\left(S_{j}\right)\right| & \begin{array}{c}k \in \mathbb{N}, a_{1}, \ldots, a_{k} \in \mathbb{R} \backslash\{0\}, S_{1}, \ldots, S_{k} \text { are } d- \\ \text { fold products of oriented closed connected surfaces, } \\ f_{1}: S_{1} \rightarrow X, \ldots, f_{k}: S_{k} \rightarrow X \text { continuous with } \\ \sum_{j=1}^{k} a_{j} \cdot H_{2 \cdot d}\left(f_{j} ; \mathbb{R}\right)\left[S_{j}\right]_{\mathbb{R}}=\alpha\end{array}\end{array}\right\}$.

In other words, $|\cdot| s$ measures the size of homology classes in terms of products of surfaces. In general, this functorial seminorm is not finite [13] because not every homology class in even degree can be represented by a product of surfaces.

Proposition 2.4 (The surface seminorm is the $\ell^{1}$-seminorm in degree 2) Let $X$ be a topological space, and let $\alpha \in H_{2}(X ; \mathbb{R})$. Then

$$
\|\alpha\|_{1}=2 \cdot|\alpha|_{S} .
$$

Proof This follows from a result of Barge and Ghys [4, Proposition 1.9] (notice that their argument applies only to classes that do not need summands represented by $S^{2}$ [4, proof of Lemme 1.7]; however, we can safely ignore these summands as they do not contribute to the $\ell^{1}$-seminorm). 
Question 2.5 Does the surface seminorm vanish on homology classes (of nonzero degree) of simply connected spaces?

Classical arguments from algebraic topology show that this is indeed true in degrees 2 and 4 (see Proposition 4.5); however, the question is open in high degrees.

Similarly to the surface seminorm $|\cdot| S$, we can also define functorial seminorms by looking at domination by eg hyperbolic manifolds (Example 4.6).

An interesting aspect of functorial seminorms is that suitable functorial seminorms give a systematic way to deduce degree theorems for maps between manifolds:

Remark 2.6 (Degree theorems) If $|\cdot|$ is a functorial seminorm on singular homology, then by definition we have, for all continuous maps $f: M \rightarrow N$ of oriented closed connected manifolds of the same dimension, the estimate

$$
|\operatorname{deg} f| \cdot\left|[N]_{\mathbb{R}}\right| \leq\left|[M]_{\mathbb{R}}\right|
$$

hence, if $\left|[N]_{\mathbb{R}}\right| \neq 0$, then we obtain the restriction

$$
|\operatorname{deg} f| \leq \frac{\left|[M]_{\mathbb{R}}\right|}{\left|[N]_{\mathbb{R}}\right|}
$$

on the mapping degree. Such restrictions are particularly interesting when there are at least for certain classes of (Riemannian) manifolds — estimates of $\left|[\cdot]_{\mathbb{R}}\right|$ in terms of the Riemannian volume or other geometric invariants.

For example, powerful degree theorems have been obtained by the use of simplicial volume and its variations (see [10, Section $0.5 ; 16$, Section 1.2]).

Conversely, in the following sections, we will translate knowledge about mapping degrees into constructions of functorial seminorms with specific properties.

\section{Representing homology classes by manifolds}

As mentioned in the introduction, one of our main tools is to represent singular homology classes by manifolds. For the sake of completeness, we recall the following classical result: 
Theorem 3.1 Let $X$ be a connected $C W$-complex, let $d \in \mathbb{N}$ and let $\alpha \in H_{d}(X ; \mathbb{Q})$ be a singular homology class.

(1) Then there exists an $a \in \mathbb{Q} \backslash\{0\}$ and an oriented closed connected $d$-dimensional smooth manifold $M$ together with a continuous map $f: M \rightarrow X$ such that

$$
a \cdot H_{d}(f ; \mathbb{Q})[M]_{\mathbb{Q}}=\alpha,
$$

where $[M]_{\mathbb{Q}} \in H_{d}(M ; \mathbb{Q})$ is the rational fundamental class of $M$.

(2) If $X$ is homotopy equivalent to a $C W$-complex with finite 2 -skeleton and $d \geq 4$, then there exists an $a \in \mathbb{Q} \backslash\{0\}$ and an oriented closed connected $d$-dimensional manifold $M$ together with a continuous map $f: M \rightarrow X$ such that

$$
a \cdot H_{d}(f ; \mathbb{Q})[M]_{\mathbb{Q}}=\alpha
$$

and such that in addition $\pi_{1}(f): \pi_{1}(M) \rightarrow \pi_{1}(X)$ is an isomorphism.

Proof The first part is a classical result by Thom [23].

For the second statement, we apply surgery theory as in Kreck [14]. Using that notation, let $B:=X \times \mathrm{BSO}$, where BSO is the classifying space of the stable special orthogonal group and let $B \rightarrow \mathrm{BO}$ be the fibration given by projection to $\mathrm{BSO}$ and the canonical covering $\mathrm{BSO} \rightarrow \mathrm{BO}$.

Given an oriented closed connected smooth manifold $\bar{v}: M \rightarrow$ BSO and a map $f: M \rightarrow X$, we obtain the $B$-manifold $f \times \bar{v}: M \rightarrow X \times \mathrm{BSO}=B$. Hence, there is an oriented bordism $F: W \rightarrow X$ over $X$ from $f: M \rightarrow X$ to a map $g: N \rightarrow X$ such that $g$ is a 2-equivalence [14, Proposition 4]; in particular, $g$ induces an isomorphism on fundamental groups. A straightforward computation in singular homology shows

$$
H_{*}(g ; \mathbb{Q})[N]_{\mathbb{Q}}=H_{*}(f ; \mathbb{Q})[M]_{\mathbb{Q}}-H_{*}(F ; \mathbb{Q})[\partial W]_{\mathbb{Q}}=H_{*}(f ; \mathbb{Q})[M]_{\mathbb{Q}} ;
$$

choosing $f$ as provided by part (1) finishes the proof.

We next extend Theorem 3.1 to general path-connected spaces and to homology classes in $H_{*}(\cdot ; \mathbb{R})$ which lie in the image of the change of coefficients homomorphism $H_{*}(\cdot ; \mathbb{Q}) \rightarrow H_{*}(\cdot ; \mathbb{R})$. Such classes are called rational, and by the universal coefficient theorem, every class in $H_{*}(\cdot ; \mathbb{R})$ is a finite $\mathbb{R}$-linear combination of rational classes. 
Corollary 3.2 Let $X$ be a path-connected topological space, let $d \in \mathbb{N}$, and let $\alpha \in H_{d}(X ; \mathbb{R})$ be rational.

(1) There is an $a \in \mathbb{Q} \backslash\{0\}$ and an oriented closed connected smooth $d$-manifold $M$ and a continuous map $f: M \rightarrow X$ such that $a \cdot H_{d}(f ; \mathbb{R})[M]_{\mathbb{R}}=\alpha$, where $[M]_{\mathbb{R}} \in H_{d}(M ; \mathbb{R})$ is the real fundamental class of $M$.

(2) If $X$ is simply connected and $d \geq 4$, then there exist $a \in \mathbb{Q} \backslash\{0\}$, an oriented closed simply connected smooth $d$-manifold $M$ and a continuous map $f: M \rightarrow X$ with $a \cdot H_{d}(f ; \mathbb{R})[M]_{\mathbb{R}}=\alpha$.

Proof Out of the combinatorial data of a singular cycle in $C_{d}(X ; \mathbb{R})$ representing $\alpha$ we can construct a connected finite CW-complex $X^{\prime}$, a rational homology class $\alpha^{\prime} \in$ $H_{d}\left(X^{\prime} ; \mathbb{R}\right)$ and a continuous map $f^{\prime}: X^{\prime} \rightarrow X$ such that $H_{d}\left(f^{\prime} ; \mathbb{R}\right)\left(\alpha^{\prime}\right)=\alpha$; if $X$ is simply connected, then we can also assume that $X^{\prime}$ is simply connected. Now the claim easily follows from the universal coefficient theorem and the previous theorem.

\section{Generating functorial seminorms via special spaces}

Every functorial seminorm on singular homology induces by restriction a functorial seminorm on the top homology of oriented closed connected manifolds. Conversely, examples of functorial seminorms on singular homology can be generated by extending functorial seminorms on the top homology of oriented closed connected manifolds (of a given dimension):

Definition 4.1 (Associated seminorm) Let $d \in \mathbb{N}$, let $\operatorname{Mfd}_{d}$ denote the class of all oriented closed connected $d$-manifolds, and let $S \subset \operatorname{Mfd}_{d}$ be a subclass.

- A functorial seminorm on fundamental classes of oriented closed connected $d$-manifolds in $S$, or briefly a functorial $S$-seminorm, is a map $v: S \rightarrow[0, \infty]$ such that

$$
|\operatorname{deg} f| \cdot v(N) \leq v(M)
$$

holds for all continuous maps $f: M \rightarrow N$ with $N, M \in S$.

If $S=\operatorname{Mfd}_{d}$, then we call such a $v$ a functorial seminorm on fundamental classes of oriented closed connected $d$-manifolds, briefly a functorial $\mathrm{Mfd}_{d}$-seminorm.

- Let $v$ be an $S$-functorial seminorm. The associated seminorm $|\cdot|$ on singular homology in degree $d$ is defined as follows. For a topological space $X$ and a homology class $\alpha \in H_{d}(X ; \mathbb{R})$ we set

$$
|\alpha|:=\inf \left\{\begin{array}{l|l}
\sum_{j=1}^{k}\left|a_{j}\right| \cdot v\left(M_{j}\right) & \begin{array}{l}
k \in \mathbb{N}, a_{1}, \ldots, a_{k} \in \mathbb{R} \backslash\{0\}, M_{1}, \ldots, M_{k} \in S, \\
f_{1}: M_{1} \rightarrow X, \ldots, f_{k}: M_{k} \rightarrow X \text { continuous } \\
\text { with } \sum_{j=1}^{k} a_{j} \cdot H_{d}\left(f_{j} ; \mathbb{R}\right)\left[M_{j}\right]_{\mathbb{R}}=\alpha
\end{array}
\end{array}\right\} ;
$$


we use the conventions $r \cdot \infty:=\infty$ for all $r \in \mathbb{R}_{>0}$ and $\inf \varnothing:=\infty$.

Theorem 4.2 (Generating functorial seminorms) Let $d \in \mathbb{N}$, let $S \subset \operatorname{Mfd}_{d}$ be a subclass and let $|\cdot|$ be the seminorm associated with a functorial seminorm $v: S \rightarrow$ $[0, \infty]$ on fundamental classes of oriented closed connected $d$-manifolds in $S$ (see Definition 4.1).

(1) Then $|\cdot|$ is a functorial seminorm on singular homology in degree $d$, and for all oriented closed connected $d$-manifolds $M$ in $S$ we have

$$
\left|[M]_{\mathbb{R}}\right|=v(M) .
$$

(2) If $S=\operatorname{Mfd}_{d}$ and $v$ is finite, then so is $|\cdot|$.

(3) The associated seminorm $|\cdot|$ is maximal in the following sense. If $|\cdot|^{\prime}$ is a functorial seminorm on singular homology in degree $d$ that extends $v$, then $|\cdot|^{\prime} \leq|\cdot|$.

Proof A straightforward computation shows that $|\cdot|$ as defined in Definition 4.1 is indeed a functorial seminorm in degree $d$. If $M$ is an oriented closed connected $d-$ manifold in $S$, then representing $[M]_{\mathbb{R}}$ by $\operatorname{id}_{M}: M \rightarrow M$ shows that $\left|[M]_{\mathbb{R}}\right| \leq v(M)$. On the other hand, $v(M) \leq\left|[M]_{\mathbb{R}}\right|$ as we now show. Let

$$
[M]_{\mathbb{R}}=\sum_{j=1}^{k} a_{j} \cdot H_{d}\left(f_{j} ; \mathbb{R}\right)\left[M_{j}\right]_{\mathbb{R}}=\sum_{j=1}^{k} a_{j} \cdot \operatorname{deg} f_{j} \cdot[M]_{\mathbb{R}}
$$

be a representation of $[M]_{\mathbb{R}}$ as in Definition 4.1 ; then $1=\sum_{j=1}^{k} a_{j} \cdot \operatorname{deg} f_{j}$ and hence

$$
v(M) \leq \sum_{j=1}^{k}\left|a_{j}\right| \cdot\left|\operatorname{deg} f_{j}\right| \cdot v(M) \leq \sum_{j=1}^{k}\left|a_{j}\right| \cdot v\left(M_{j}\right)
$$

by functoriality of $v$ on $S$. This proves the first part.

The second part follows from the fact that every real singular homology class of a pathconnected space is an $\mathbb{R}$-linear combination of rational classes, which can - up to a nonzero factor - be represented by oriented closed connected manifolds (Corollary 3.2).

The last part follows directly from the construction of $|\cdot|$, the triangle inequality and the definition of functoriality.

For example, the $\ell^{1}$-seminorm can be viewed as the functorial seminorm generated by simplicial volume: 
Proposition 4.3 Let $d \in \mathbb{N} \backslash\{3\}$. Then on the category of connected finite $C W-$ complexes the functorial seminorm on singular homology in degree $d$ associated with the simplicial volume in dimension $d$ coincides with the $\ell^{1}$-seminorm in degree $d$.

Proof Clearly, the statement holds in degree 0 . In degree 1 the claim follows directly from the Hurewicz theorem.

In degree 2, one has to understand the simplicial volume of surfaces and how singular homology classes in degree 2 can be represented by surfaces. If $S$ is an oriented closed connected surface of genus $g \geq 1$, then [10, page 9; 5, Proposition C.4.7]

$$
\|S\|=4 \cdot g-4=2 \cdot|\chi(S)|
$$

combining this fact with Proposition 2.4 proves the claim in degree 2.

Suppose now that the degree $d$ is at least 4. In view of Theorem 4.2(3), the functorial seminorm $|\cdot|$ associated with the simplicial volume satisfies $|\cdot| \geq\|\cdot\|_{1}$; thus, it suffices to prove the reverse inequality.

Let $X$ be a connected finite CW-complex, and let $\alpha \in H_{d}(X ; \mathbb{R})$. We can write $\alpha=$ $\sum_{j=1}^{k} a_{j} \cdot \alpha_{j}$, where $\alpha_{1}, \ldots, \alpha_{k} \in H_{d}(X ; \mathbb{R})$ are rational and $a_{1}, \ldots, a_{n} \in \mathbb{R}$. For $n \in \mathbb{N}$ we let $\alpha^{(n)}:=\sum_{j=1}^{k} a_{j}^{(n)} \cdot \alpha_{j}$, where $\left(a_{j}^{(n)}\right)_{n \in \mathbb{N}}$ is a sequence in $\mathbb{Q}$ that approximates $a_{j}$; by construction, the $\alpha^{(n)}$ are rational and the triangle inequality shows that

$$
\lim _{n \rightarrow \infty}\left|\alpha^{(n)}-\alpha\right|=0 \quad \text { and } \quad \lim _{n \rightarrow \infty}\left\|\alpha^{(n)}-\alpha\right\|_{1}=0 .
$$

Therefore, it suffices to prove $|\cdot| \leq\|\cdot\|_{1}$ for rational classes in $H_{d}(X ; \mathbb{R})$.

If $\alpha$ is rational, then by Theorem 3.1, there is an $a \in \mathbb{R} \backslash\{0\}$, and a continuous map $f: M \rightarrow X$ from some oriented closed connected $d$-manifold $M$ such that

$$
a \cdot H_{d}(f ; \mathbb{R})[M]_{\mathbb{R}}=\alpha
$$

and such that in addition $\pi_{1}(f): \pi_{1}(M) \rightarrow \pi_{1}(X)$ is an isomorphism. Applying the mapping theorem in bounded cohomology [10, Section 3.1; 12, Theorem 4.3] (combined with the duality principle for the $\ell^{1}$-seminorm [10, Corollary on page 17]) shows that $H_{d}(f ; \mathbb{R}): H_{d}(M ; \mathbb{R}) \rightarrow H_{d}(X ; \mathbb{R})$ is isometric with respect to the $\ell^{1}$-seminorm. In particular,

$$
\|\alpha\|_{1}=|a| \cdot\left\|[M]_{\mathbb{R}}\right\|_{1}=|a| \cdot\|M\| \geq|\alpha| .
$$

The surface seminorm is also a seminorm defined as in Definition 4.1: 
Proposition 4.4 Let $d \in \mathbb{N}$, let $S \subset \operatorname{Mfd}_{2 \cdot d}$ be the subclass of products of $d$ oriented closed connected surfaces, and let

$$
v: S \rightarrow[0, \infty], \quad M \mapsto|\chi(M)| .
$$

Then $v$ is a functorial seminorm on fundamental classes of oriented closed connected manifolds in $S$, and the functorial seminorm on $H_{2 \cdot d}(\cdot ; \mathbb{R})$ associated with $v$ is the surface seminorm of Example 2.3.

Proof That $v$ indeed is functorial can, for example, be seen via the simplicial volume, the proportionality principle for simplicial volume and the multiplicativity of the Euler characteristic [11, page 303; 15, Corollary 6.5].

That the seminorm associated with $v$ and the surface seminorm coincide follows directly from the definitions.

Proposition 4.5 The surface seminorm $|\cdot| S$ vanishes on all singular homology classes of simply connected spaces of degree 2 or 4 .

Proof Let $X$ be a simply connected topological space, and let $\alpha \in H_{*}(X ; \mathbb{R})$ be a homology class of degree 2 or 4 .

If $\alpha$ is of degree 2, then - because $X$ is simply connected - we have an isomorphism $H_{2}(X ; \mathbb{Z}) \cong \pi_{2}(X)$. Hence every integral homology class in degree 2 is represented by a map from the sphere $S^{2}$. Using the universal coefficient theorem and the fact that $S^{2}$ admits self-maps of arbitrarily large degree, it follows that the surface seminorm vanishes on $\mathrm{H}_{2}(X ; \mathbb{R})$.

Let $\alpha$ now be of degree 4 . In view of the triangle inequality, we can assume without loss of generality that $\alpha$ is rational. Then by Corollary 3.2 we can represent $\alpha$ as

$$
a \cdot H_{d}(f ; \mathbb{R})[M]_{\mathbb{R}}=\alpha,
$$

where $M$ is an oriented closed simply connected 4 -manifold, $f: M \rightarrow X$ is a continuous map, and $a \in \mathbb{R} \backslash\{0\}$. Moreover, the simply connected 4-manifold $M$ is dominated by a product $S^{1} \times S^{1} \times S$, where $S$ is a suitable oriented closed connected surface [13, Proposition 7.1]. Because $\chi\left(S^{1} \times S^{1} \times S\right)=0$ it follows that $|\alpha|_{S}=0$.

Similarly to the definition of the surface seminorm, we can also take hyperbolic manifolds as building blocks of a functorial seminorm: 
Example 4.6 (The hyperbolic seminorm) Let $d \in \mathbb{N}$, let $H \subset \operatorname{Mfd}_{d}$ be the subclass of all oriented closed connected smooth $d$-manifolds that admit a hyperbolic Riemannian metric. Then

$$
v: H \rightarrow[0, \infty], \quad M \mapsto \operatorname{vol}(M)
$$

is well defined and functorial (because the volume of hyperbolic manifolds can be expressed in terms of the simplicial volume [10, Section $0.3 ; 24$, Theorem 6.2] and because the simplicial volume is functorial).

We point out that it is still an open problem whether every manifold can be dominated by a hyperbolic manifold [13, Conjecture 7.2]; so it is not known whether the functorial seminorm on $H_{d}(\cdot ; \mathbb{R})$ associated with $v$ is finite.

Remark 4.7 (Generating functorial seminorms via Poincaré spaces) Recall that a $\mathbb{Q}$-Poincaré space of formal dimension $d$ is a connected CW-complex $X$ together with a homology class $[X] \in H_{d}(X ; \mathbb{Q})$, the fundamental class, such that

$$
\cdot \cap[X]: H^{*}(X ; \mathbb{Q}) \rightarrow H_{*-d}(X ; \mathbb{Q})
$$

is an isomorphism. In particular, one can introduce the notion of mapping degree for continuous maps between $\mathbb{Q}$-Poincaré spaces of the same formal dimension.

Similarly to Definition 4.1 and Theorem 4.2, any functorial seminorm on the fundamental classes of $\mathbb{Q}$-Poincaré complexes of a given dimension gives rise to an associated functorial seminorm on singular homology in the given degree.

\section{Functorial seminorms which are (not) induced from the singular chain complex}

One source of functorial seminorms on singular homology is the class of functorial seminorms on the singular chain complex. Let $d \in \mathbb{N}$. A functorial seminorm on the singular chain complex in degree $d$ consists of a choice of a seminorm $|\cdot|$ on $C_{d}(X ; \mathbb{R})$ for every topological space $X$ such that the following "functoriality" holds: for all continuous maps $f: X \rightarrow Y$ between topological spaces and all $c \in C_{d}(X ; \mathbb{R})$,

$$
\left|C_{d}(f ; \mathbb{R})(c)\right| \leq|c| .
$$

Such a functorial seminorm on the singular chain complex is finite if all the seminorms involved are finite seminorms. For example, the $\ell^{1}$-norm on the chain level (Example 2.2) is a finite functorial seminorm on the singular chain complex. 
(Non)example 5.1 ( $\ell^{p}$-seminorms) Let $d \in \mathbb{N}$, let $p \in(1, \infty]$, and let $|\cdot|_{p}$ be the $p$-norm on $C_{d}(\cdot ; \mathbb{R})$ with respect to the (unordered) basis given by the set of all singular $d$-simplices. Then $|\cdot|_{p}$ is not a functorial seminorm on the singular chain complex in degree $d$.

We consider $X:=\{x, y\}$ with the discrete topology and $f: X \rightarrow X$ mapping both points to $x$. Let $c:=\sigma_{x}+\sigma_{y} \in C_{d}(X ; \mathbb{R})$, where $\sigma_{x}$ and $\sigma_{y}$ are the constant singular $d$-simplices mapping to $x$ and $y$ respectively. Then

$$
\left|C_{d}(f ; \mathbb{R})(c)\right|_{\infty}=\left|2 \cdot \sigma_{x}\right|_{\infty}=2>1=\left|\sigma_{x}+\sigma_{y}\right|_{\infty}=|c|_{\infty},
$$

and for $p \in(1, \infty)$ we obtain

$$
\left|C_{d}(f ; \mathbb{R})(c)\right|_{p}=\left|2 \cdot \sigma_{x}\right|_{p}=2>\sqrt[p]{1^{p}+1^{p}}=\left|\sigma_{x}+\sigma_{y}\right|_{p}=|c|_{p} .
$$

Hence $|\cdot|_{p}$ is not functorial.

Clearly, any (finite) functorial seminorm on the singular chain complex in degree $d$ induces a (finite) functorial seminorm on singular homology in degree $d$ by taking the infimum of the seminorms of cycles representing a given class. Notice that being induced from a finite functorial seminorm on the singular chain complex is a rather strong condition:

Proposition 5.2 Let $d \in \mathbb{N}$ and let $|\cdot|$ be a finite functorial seminorm on the singular chain complex in degree $d$. Then

$$
|\cdot| \leq\left|\operatorname{id}_{\Delta^{d}}\right| \cdot\|\cdot\|_{1} .
$$

Proof Let $c=\sum_{j=0}^{k} a_{j} \cdot \sigma_{j} \in C_{d}(X ; \mathbb{R})$ be a singular chain (in reduced form). Viewing $\operatorname{id}_{\Delta^{d}}: \Delta^{d} \rightarrow \Delta^{d}$ as a singular $d$-simplex on $\Delta^{d}$, functoriality of $|\cdot|$ yields

$$
|c| \leq \sum_{j=0}^{k}\left|a_{j}\right| \cdot\left|\sigma_{j} \circ \mathrm{id}_{\Delta^{d}}\right| \leq \sum_{j=0}^{k}\left|a_{j}\right| \cdot\left|\operatorname{id}_{\Delta^{d}}\right|=\left|\operatorname{id}_{\Delta^{d}}\right| \cdot\|c\|_{1},
$$

as desired.

Corollary 5.3 In particular, because the $\ell^{1}$-seminorm is trivial on simply connected spaces [10, Section 3.1; 12, Theorem 2.4], every functorial seminorm on singular homology induced from a finite functorial seminorm on the singular chain complex is trivial on simply connected spaces.

Concerning the converse question "Which (finite) functorial seminorms on singular homology are induced from (finite) functorial seminorms on the singular chain complex?", we prove the following. 
- Every functorial seminorm on singular homology is induced from some (in general infinite) functorial seminorm on the singular chain complex (Proposition 5.4).

- There exist finite functorial seminorms on singular homology that are not induced from a finite functorial seminorm on the singular chain complex (Theorem 5.7). Therefore, Corollary 5.3 is not strong enough to answer Gromov's question (Question 1.1(2)) in the positive for all finite functorial seminorms.

Proposition 5.4 Let $d \in \mathbb{N}$, and let $|\cdot|$ be a functorial seminorm on singular homology in degree $d$. Then there is a functorial seminorm $|\cdot|$ on the singular chain complex in degree $d$ inducing $|\cdot|$, ie for all topological spaces $X$ and all $\alpha \in H_{d}(X ; \mathbb{R})$ we have

$$
|\alpha|=\inf \left\{|c| \mid c \in C_{d}(X ; \mathbb{R}) \text { is a cycle representing } \alpha\right\} .
$$

Proof Let $X$ be a topological space. We denote by $i: Z_{d}(X ; \mathbb{R}) \rightarrow C_{d}(X ; \mathbb{R})$ and $p: Z_{d}(X ; \mathbb{R}) \rightarrow H_{d}(X ; \mathbb{R})$ the inclusion of the $d$-cycles and the projection onto the $d^{\text {th }}$ homology group respectively. Define a seminorm $|\cdot|$ on $C_{d}(X ; \mathbb{R})$ by setting

$$
|\cdot|:=i_{*} p^{*}|\cdot|
$$

where $i_{*}$ and $p^{*}$ are defined as follows.

(1) Construction of $p^{*}|\cdot|$ Let $p: V \rightarrow U$ be a surjective homomorphism of $\mathbb{R}$-vector spaces, and let $|\cdot|$ be a seminorm on $U$. Then

$$
p^{*}|\cdot|: V \rightarrow[0, \infty], \quad x \mapsto|p(x)|
$$

is a seminorm on $V$ (this is a straightforward calculation).

(2) Construction of $i_{*}|\cdot|$ Let $i: U \rightarrow V$ be the inclusion of a subspace of an $\mathbb{R}$-vector space, and let $|\cdot|$ be a seminorm on $U$. Then

$$
i_{*}|\cdot|: V \rightarrow[0, \infty], \quad x \mapsto \begin{cases}|x| & \text { if } x \in U, \\ \infty & \text { if } x \in V \backslash U,\end{cases}
$$

is a seminorm on $V$; clearly, $i_{*}|0|=|0|=0$, and $i_{*}|\cdot|$ is compatible with scalar multiplication. Moreover, the triangle inequality is satisfied. Let $x, y \in V$. If $x \in V \backslash U$ or $y \in V \backslash U$, then $i_{*}|x|=\infty$ or $i_{*}|y|=\infty$, so that the triangle inequality is trivially satisfied. The only remaining case is that $x, y \in U$, and in this case the triangle inequality is satisfied, because $|\cdot|$ is a seminorm on $U$. Note that if $U \neq V$, then $i_{*}|\cdot|$ is infinite.

Why is $|\cdot|=i_{*} p^{*}|\cdot|$ functorial? Let $f: X \rightarrow Y$ be a continuous map and let $c \in C_{d}(X ; \mathbb{R})$. If $c$ is not a cycle, then $|c|=\infty$, and so $\left|C_{d}(f ; \mathbb{R})(c)\right| \leq|c|$. In case $c$ is a cycle, then $C_{d}(f ; \mathbb{R})(c)$ is a cycle as well and thus

$$
\left|C_{d}(f ; \mathbb{R})(c)\right|=\left|\left[C_{d}(f ; \mathbb{R})(c)\right]\right|=\left|H_{d}(f ; \mathbb{R})[c]\right| \leq|[c]|=|c|
$$


because $|\cdot|$ is functorial.

Moreover, $|\cdot|$ induces $|\cdot|$ on homology because for all cycles $c$ we have $|[c]|=|c|$ by construction of $|\cdot|$.

However, even if the given functorial seminorm on singular homology is finite, the corresponding functorial seminorm on the singular chain complex provided in the proof of Proposition 5.4 is not finite. This is not merely an artefact of this construction: in the following we give an example of a finite functorial seminorm on singular homology that grows too fast (compared to the $\ell^{1}$-seminorm) to be induced from a finite functorial seminorm on the singular chain complex.

Definition 5.5 (Degree monotonic map) A function $\varphi: \mathbb{R}_{\geq 0} \rightarrow \mathbb{R}_{\geq 0}$ that is monotonically growing is called degree monotonic if for all $x \in \mathbb{R}_{\geq 0}$ and all $d \in \mathbb{N}$,

$$
\varphi(d \cdot x) \geq d \cdot \varphi(x) .
$$

Proposition 5.6 Let $d \in \mathbb{N}$ and let $v: \operatorname{Mfd}_{d} \rightarrow \mathbb{R}_{\geq 0}$ be a finite functorial seminorm on fundamental classes of oriented closed connected $d$-manifolds. If $\varphi: \mathbb{R}_{\geq 0} \rightarrow \mathbb{R}_{\geq 0}$ is a degree monotonic map, then the composition

$$
\varphi \circ v: \operatorname{Mfd}_{d} \rightarrow \mathbb{R}_{\geq 0}
$$

is a finite functorial seminorm on fundamental classes of oriented closed connected $d$-manifolds.

Proof For all continuous maps $f: M \rightarrow N$ between oriented closed connected $d$-manifolds, we have $v(M) \geq|\operatorname{deg} f| \cdot v(N)$, and thus

$$
\varphi \circ v(M) \geq \varphi(|\operatorname{deg} f| \cdot v(N)) \geq|\operatorname{deg} f| \cdot \varphi \circ v(N)
$$

by the degree monotonicity of $\varphi$.

Theorem 5.7 There are finite functorial seminorms on singular homology that are not induced from a finite functorial seminorm on the singular chain complex.

Proof Let $\varphi: \mathbb{R}_{\geq 0} \rightarrow \mathbb{R}_{\geq 0}$ be a degree monotonic map that grows faster than linearly in the sense that $\lim _{x \rightarrow \infty} \varphi(x) / x=\infty$; for instance, for every $a \in \mathbb{R}_{>1}$ the map

$$
\mathbb{R}_{\geq 0} \rightarrow \mathbb{R}_{\geq 0}, \quad x \mapsto x^{a},
$$

has this property. Moreover, let $d \in \mathbb{N}_{\geq 2}$. 
We now consider the functorial seminorm $|\cdot|$ on singular homology in degree $d$ associated with the finite functorial seminorm on fundamental classes of oriented closed connected $d$-manifolds given by composing $\varphi$ with the simplicial volume (Proposition 5.6 and Theorem 4.2); notice that $|\cdot|$ is finite.

Assume for a contradiction that $|\cdot|$ were induced from a finite functorial seminorm. Then in view of Proposition 5.2 we would have

$$
|\cdot| \leq\left|\mathrm{id}_{\Delta^{d}}\right| \cdot\|\cdot\|_{1}
$$

However, we now show that $|\cdot|$ "grows too fast" to be able to satisfy this estimate. To see this consider the properties of hyperbolic manifolds more closely. Let $M$ be an oriented closed connected hyperbolic $d$-manifold. Then the fundamental group $\pi_{1}(M)$ of $M$ is residually finite [20, page 542]; so for any $k \in \mathbb{N}$ there is a subgroup $\Gamma_{k} \subset \pi_{1}(M)$ satisfying

$$
k \leq\left[\pi_{1}(M): \Gamma_{k}\right]<\infty .
$$

For $k \in \mathbb{N}$ we let $p_{k}: M_{k} \rightarrow M$ denote the covering associated with the inclusion $\Gamma_{k} \subset$ $\pi_{1}(M)$; hence, $M_{k}$ also is an oriented closed connected (hyperbolic) $d$-manifold and

$$
\left|\operatorname{deg} p_{k}\right|=\left[\pi_{1}(M): \Gamma_{k}\right] \geq k \text {. }
$$

Because $M$ is hyperbolic, the simplicial volume $\|M\|$ is nonzero [10, Section $0.3 ; 24$, Theorem 6.2]; thus, $\left\|M_{k}\right\| \geq k \cdot\|M\|$ tends to $\infty$ for $k \rightarrow \infty$. By definition, $\varphi$ grows faster than linearly and so

$$
\frac{\left|\left[M_{k}\right]_{\mathbb{R}}\right|}{\left\|\left[M_{k}\right]_{\mathbb{R}}\right\|_{1}}=\frac{\varphi\left(\left\|M_{k}\right\|\right)}{\left\|M_{k}\right\|}
$$

tends to $\infty$ for $k \rightarrow \infty$, contradicting the estimate in (1). Therefore, the finite functorial seminorm $|\cdot|$ on singular homology is not induced from a finite functorial seminorm on the singular chain complex.

Question 5.8 In light of the example constructed in the proof of Theorem 5.7, it is natural to ask for a reasonable notion of equivalence of functorial seminorms on singular homology or for a notion of domination of one functorial seminorm by another. Is the $\ell^{1}$-seminorm on singular homology "maximal" among finite functorial seminorms on singular homology with respect to such a notion? (This should also be compared with Proposition 7.6.) 


\section{6 (In)flexible manifolds}

The constructions of interesting functorial seminorms in Section 7.1 below require as input simply connected manifolds that are inflexible; recall that an oriented closed connected manifold $M$ is inflexible if it admits only self-maps of degree 0,1 or -1 , ie $\operatorname{deg}(M, M) \subset\{-1,0,1\}$.

Remark 6.1 Looking at iterated compositions shows that an oriented closed connected manifold $M$ is flexible if and only if $|\operatorname{deg}(M, M)|=\infty$. Conversely, the manifold $M$ is inflexible if and only if $\operatorname{deg}(M, M)$ is finite.

Remark 6.2 If a manifold is flexible, then — by functoriality - its simplicial volume is zero. In particular, oriented closed connected hyperbolic manifolds are inflexible, as they have nonzero simplicial volume. However, for simply connected manifolds the simplicial volume is zero and hence the simplicial volume cannot serve as an obstruction to flexibility in this case.

In this section we show how rational homotopy theory and surgery allow one to construct examples of simply connected inflexible manifolds, building upon examples of Arkowitz and Lupton [2] (Sections 6.1 and 6.2). We briefly discuss strongly inflexible manifolds in Section 6.3. Finally, in Section 6.4, we discuss the class of simply connected flexible manifolds from the viewpoint of rational homotopy theory. To make this section more readable we have moved most of the calculations with differential graded algebras and the proof of inheritance properties of simply connected inflexible manifolds to Appendices I and II.

\section{1 (In)flexibility and rational homotopy theory}

We start by giving an overview of the construction of simply connected inflexible manifolds and introducing key notation and definitions.

Rational homotopy theory provides the rationalisation functor $\cdot \mathbb{Q}$ on the category of simply connected spaces and an equivalence of categories between the category of simply connected rational spaces and the category of certain differential graded algebras, the so-called minimal models. For the basic definitions in rational homotopy theory, we refer to the book by Félix, Halperin and Thomas [7].

More concretely, if $M$ is an oriented closed simply connected manifold, then the associated minimal model $A_{M}$ is a differential graded algebra over $\mathbb{Q}$ whose cohomology coincides with the rational cohomology of $M$; in particular, $A_{M}$ has a cohomological fundamental class $\left[A_{M}\right]$, namely the cohomology class of $H^{*}\left(A_{M}\right) \cong H^{*}(M ; \mathbb{Q})$ dual to the fundamental class $[M]_{\mathbb{Q}}$ of $M$. 
Any self-map $f: M \rightarrow M$ induces a corresponding dga endomorphism $A_{f}: A_{M} \rightarrow$ $A_{M}$; using the cohomological fundamental class $\left[A_{M}\right]$ of $A_{M}$ we can associate a mapping degree to $A_{f}$, and this mapping degree coincides with $\operatorname{deg}(f)$. In particular, if $A_{M}$ is "inflexible", as defined in Definition 6.5 below, then so is $M$.

Hence, it suffices to find differential graded algebras that are minimal models of simply connected manifolds and whose cohomological fundamental class is inflexible; notice that the latter condition is algebraic by definition and moreover that Theorem 6.11 below entails that this is also true of the former condition.

We now give a precise definition of inflexibility and duality in the world of differential graded algebras:

Definition 6.3 ((In)flexible (co)homology classes) - A homology class $\alpha \in$ $H_{*}(X ; \mathbb{Q})$ of a topological space $X$ is called flexible if there is a continuous map $f: X \rightarrow X$ such that

$$
H_{*}(f ; \mathbb{Q})(\alpha)=d \cdot \alpha
$$

for some $d \in \mathbb{Q} \backslash\{-1,0,1\}$. A homology class is called inflexible if it is not flexible.

(In particular, an oriented closed connected manifold is inflexible if and only if its fundamental class is inflexible).

- A cohomology class $\alpha \in H^{*}(A)$ of a differential graded algebra $A$ is flexible if there is a dga endomorphism $f: A \rightarrow A$ such that

$$
H^{*}(f)(\alpha)=d \cdot \alpha
$$

for some $d \in \mathbb{Q} \backslash\{-1,0,1\}$. A cohomology class is inflexible if it is not flexible.

Definition 6.4 (Poincaré differential graded algebra) Let $n \in \mathbb{N}$. A Poincaré differential graded algebra of formal dimension $n$ is a simply connected differential graded algebra $A$ together with a cohomology class $[A] \in H^{n}(A)$, the fundamental class, satisfying the following conditions.

(1) For all $j \in \mathbb{N}_{>n}$ we have $H^{j}(A)=0$.

(2) The map

$$
\mathbb{Q} \rightarrow H^{n}(A), \quad a \mapsto a \cdot[A],
$$

is an isomorphism.

(3) For all $j \in\{0, \ldots, n\}$, the pairing $H^{j}(A) \times H^{n-j}(A) \rightarrow H^{n}(A) \cong \mathbb{Q}$ (where we use the isomorphism $H^{n}(A) \cong \mathbb{Q}$ of the previous item) given by multiplication identifies $H^{j}(A)$ with $\operatorname{Hom}_{\mathbb{Q}}\left(H^{n-j}(A), \mathbb{Q}\right)$. 
Definition 6.5 (Inflexible Poincaré algebra/space)

- A Poincare differential graded algebra $(A,[A])$ is inflexible if its fundamental class $[A]$ is inflexible in the sense of Definition 6.3.

- A $\mathbb{Q}$-Poincaré space $(X,[X])$ (see Remark 4.7 for a definition) is inflexible if its fundamental class $[X]$ is inflexible in the sense of Definition 6.3.

\subsection{Simply connected inflexible manifolds}

Arkowitz and Lupton gave examples of differential graded algebras that admit only finitely many homotopy classes of dga endomorphisms [2, Examples 5.1 and 5.2]. Moreover, they showed how to prove that these differential graded algebras are minimal models of simply connected closed manifolds. In particular, these simply connected manifolds are inflexible.

In Appendix I we review their construction, and give two more examples of differential graded algebras with inflexible fundamental class:

Theorem 6.6 There are inflexible Poincaré differential graded algebras $\left(A_{1},\left[A_{1}\right]\right)$, $\left(A_{2},\left[A_{2}\right]\right),\left(A_{3},\left[A_{3}\right]\right)$ and $\left(A_{4},\left[A_{4}\right]\right)$ of formal dimensions 64, 108, 208 and 228 respectively.

Proof This is proved in Appendix I (Corollary I.7 and Proposition I.10), where the choice of fundamental class is also specified (Proposition I.6).

In the following, we focus on the realisability of these Poincare differential graded algebras by simply connected manifolds (for simplicity, we consider only the case of trivial total Pontryagin class):

Definition 6.7 (Realisability by manifolds) Suppose $(A,[A])$ is a Poincaré differential graded algebra of formal dimension $n$. We then write $\mathcal{M}(A,[A])$ for the class of all oriented closed simply connected $n$-manifolds $M$ that have trivial total Pontryagin class and that satisfy

$$
\left(A_{M},\left[A_{M}\right]\right) \cong(A,[A])
$$

Theorem 6.8 (Simply connected inflexible manifolds) For the above Poincaré dgas $\left(A_{1},\left[A_{1}\right]\right), \ldots,\left(A_{4},\left[A_{4}\right]\right)$ the classes $\mathcal{M}\left(A_{1},\left[A_{1}\right]\right), \ldots, \mathcal{M}\left(A_{4},\left[A_{4}\right]\right)$ are nonempty. In particular, there are oriented closed simply connected inflexible manifolds of dimension 64, 108, 208, 228 respectively. 
We now assemble the statements we need to prove Theorem 6.8. As first step, we show that the differential graded algebras $A_{1}, \ldots, A_{4}$ are the corresponding dgas of rational $\mathbb{Q}$-Poincaré spaces:

Proposition 6.9 (Realisibility by $\mathbb{Q}$-Poincaré spaces) For the above Poincaré dgas $\left(A_{1},\left[A_{1}\right]\right), \ldots,\left(A_{4},\left[A_{4}\right]\right)$ there are corresponding simply connected rational $\mathbb{Q}$-Poincaré spaces $\left(X_{1},\left[X_{1}\right]\right), \ldots,\left(X_{4},\left[X_{4}\right]\right)$ respectively realising these dgas as their minimal models such that the cohomology classes corresponding to the fundamental classes $\left[A_{j}\right]$ are dual to the fundamental classes $\left[X_{j}\right]$; these spaces $X_{1}, \ldots, X_{4}$ are unique up to rational homotopy equivalence, and they have formal dimension

$$
\text { 64, 108, 208, 228, }
$$

respectively.

Proof Because the dgas $A_{1}, \ldots, A_{4}$ are Poincaré, the correspondence between rational spaces and minimal Sullivan algebras [7, Chapter 17] shows that up to rational homotopy equivalence there is a unique simply connected rational space that is a $\mathbb{Q}$ Poincaré space whose minimal model is $A_{1}, A_{2}, A_{3}$ or $A_{4}$ respectively, and whose fundamental class corresponds to the fundamental class of the respective dga.

Moreover, there is a formula expressing the formal dimension in terms of the degrees of the generators of an elliptic dga [7, Proposition 38.3]. The generators for our examples along with their degrees are given in Section I.1 and the calculation boils down to the formal dimension

$$
\left|y_{1}\right|+\left|y_{2}\right|+\left|y_{3}\right|+|z|-\left(\left|x_{1}\right|-1\right)-\left(\left|x_{2}\right|-1\right),
$$

and hence to the formal dimensions 64, 108, 208 and 228 respectively.

Corollary 6.10 (Inflexible $\mathbb{Q}$-Poincaré spaces) In particular, the simply connected rational $\mathbb{Q}$-Poincaré spaces $\left(X_{1},\left[X_{1}\right]\right), \ldots,\left(X_{4},\left[X_{4}\right]\right)$ from Proposition 6.9 are inflexible.

Proof Let $j \in\{1, \ldots, 4\}$. Assume for a contradiction that $X_{j}$ is flexible. Then there is a continuous map $f: X_{j} \rightarrow X_{j}$ of degree $d \notin\{-1,0,1\}$. The map $f$ induces a dga morphism $A_{j} \rightarrow A_{j}$ of degree $d$, because $A_{j}$ is a minimal model of $X_{j}$. However, this contradicts inflexibility of the dga $A_{j}$ established in Proposition I.10.

It now remains to show that the rational $\mathbb{Q}$-Poincaré spaces of Corollary 6.10 can be realised by simply connected manifolds. To this end, we apply a foundational theorem of Barge [3] and Sullivan [22] (a special case is Theorem 6.11 below). This 
theorem gives necessary and sufficient conditions for a rational $\mathbb{Q}$-Poincaré space $X$ to be realised by a manifold with prescribed rational Pontryagin classes; moreover the conditions are formulated using only the rational cohomology ring of $X$. Before stating the theorem we recall some basic terminology.

Let $\lambda: H \otimes H \rightarrow \mathbb{Q}$ be a nonsingular symmetric bilinear form over a finite-dimensional $\mathbb{Q}$-vector space $H$. Recall that a Lagrangian for $(H, \lambda)$ is a subspace $L \subset H$ such that $\left.\lambda\right|_{L \times L}=0$ and $2 \cdot \operatorname{rank}(L)=\operatorname{rank}(H)$; the pair $(H, \lambda)$ is called metabolic if it admits a Lagrangian. The Witt group of $\mathbb{Q}$, denoted by $W_{0}(\mathbb{Q})$, is the Grothendieck group of the monoid of isomorphism classes of nonsingular symmetric bilinear forms on finite-dimensional $\mathbb{Q}$-vector spaces under the operation of direct sum and modulo the subgroup generated by differences of metabolic forms [17, I Section 7].

If $(X,[X])$ is a $\mathbb{Q}$-Poincaré space of formal dimension $4 k$ then the cup product followed by evaluation on $[X]$ defines a nonsingular symmetric bilinear form

$$
\left(H^{2 k}(X ; \mathbb{Q}), \lambda_{[X]}\right) .
$$

The Witt index of $(X,[X])$ is defined to be the equivalence class of this form in the Witt group of $\mathbb{Q}$ :

$$
\tau_{[X]}:=\left[H^{2 k}(X ; \mathbb{Q}), \lambda_{[X]}\right] \in W_{0}(\mathbb{Q}) .
$$

Theorem 6.11 (Realising rational $\mathbb{Q}$-Poincaré spaces by manifolds $[3$, Théorème 1 ; 22, Theorem 13.2]) Suppose that $(X,[X])$ is a rational $\mathbb{Q}$-Poincaré complex of formal dimension $4 k$ and that $p_{*} \in H^{4 *}(X ; \mathbb{Q})$ is a cohomology class with $p_{0}=$ $1 \in H^{0}(X ; \mathbb{Q})$. Then there is an oriented closed simply connected manifold $(M,[M])$ with total Pontryagin class $p_{M}$ and a rational equivalence $f: M \rightarrow X$ with

$$
H_{4 k}(f ; \mathbb{Q})\left([M]_{\mathbb{Q}}\right)=[X] \text { and } H^{4 k}(f ; \mathbb{Q})\left(p_{M}\right)=p_{*}
$$

if and only if the following two conditions hold.

(1) The Witt index $\tau_{[X]}$ of $(X,[X])$ lies in the image of the homomorphism $W_{0}(\mathbb{Z}) \rightarrow$ $W_{0}(\mathbb{Q})$.

(2) There is an equality $\operatorname{sign}(X,[X])=\left\langle L\left(p_{*}\right),[X]\right\rangle$, where $L\left(p_{*}\right)$ is the Hirzebruch $L$-class evaluated at $p_{*}$.

Proposition 6.12 (Witt index) Let $j \in\{1, \ldots, 4\}$, and let $\left(X_{j},\left[X_{j}\right]\right)$ be the corresponding $\mathbb{Q}$-Poincaré space of Proposition 6.9 (oriented by the choice of fundamental class in Proposition I.6). Then $\left(X_{j},\left[X_{j}\right]\right)$ has trivial Witt index, ie $\tau_{\left[X_{j}\right]}=0 \in W_{0}(\mathbb{Q})$. In particular, the signature $\operatorname{sign}\left(X_{j}\right)$ of $\left(X_{j},\left[X_{j}\right]\right)$ equals 0 . 
Proof The result follows by explicit computation. For example, the intersection form of $\left(A_{1},\left[x_{2}^{16}\right]\right)$ and hence $\left(X_{1},\left[X_{1}\right]\right)$ is computed in Proposition I.8 where a basis for the middle cohomology is given. With respect to this matrix, the intersection matrix is an element of $\operatorname{GL}(4, \mathbb{Z})$ and has Lagrangian with basis $\left\{\left[x_{2} w\right],\left[x_{1}^{2} w\right]\right\}$. Similar calculations prove the proposition for $A_{2}, A_{3}$ and $A_{4}$.

Proof of Theorem 6.8 Let $j \in\{1, \ldots, 4\}$, and let $\left(X_{j},\left[X_{j}\right]\right)$ be the simply connected rational $\mathbb{Q}$-Poincaré space provided by Proposition 6.9. In view of Proposition 6.12, the Witt index $\tau_{\left[X_{j}\right]}$ lies in the image of the homomorphism $W_{0}(\mathbb{Z}) \rightarrow W_{0}(\mathbb{Q})$; choosing $p:=1 \in H^{0}\left(X_{j} ; \mathbb{Q}\right) \subset H^{*}\left(X_{j} ; \mathbb{Q}\right)$, we obtain

$$
\operatorname{sign}\left(X_{j},\left[X_{j}\right]\right)=0=\langle L(p),[X]\rangle .
$$

Therefore, by Theorem 6.11 , there exists an oriented closed simply connected manifold $(M,[M])$ rationally equivalent to $\left(X_{j},\left[X_{j}\right]\right)$ with trivial Pontryagin class; because $\left(A_{j},\left[A_{j}\right]\right)$ is the minimal model of $\left(X_{j},\left[X_{j}\right]\right)$, it follows that $M \in \mathcal{M}\left(A_{j},\left[A_{j}\right]\right)$.

In particular, this manifold $M$ is inflexible (using the same arguments as in the proof of Corollary 6.10).

Remark 6.13 (Scaling the fundamental class) The results of Theorem 6.8, Propositions 6.9 and 6.12 and Corollary 6.10 all hold if the fundamental classes of the respective dgas/Poincaré complexes are scaled by any nonzero rational number. The key point is that if $\lambda$ is a nonsingular symmetric bilinear form on a finite-dimensional $\mathbb{Q}$-vector space that is trivial in the Witt group $W_{0}(\mathbb{Q})$ and if $a \in \mathbb{Q} \backslash\{0\}$, then $a \cdot \lambda$ is also trivial in the Witt group (because any Lagrangian for $\lambda$ is also a Lagrangian for $a \cdot \lambda$ ). Notice that scalars with different absolute values lead to different homotopy types of simply connected inflexible manifolds in the same rational homotopy type (Proposition II.8).

Starting with the manifolds in $\mathcal{M}\left(A_{1},\left[A_{1}\right]\right), \ldots, \mathcal{M}\left(A_{4},\left[A_{4}\right]\right)$ we can construct many more simply connected inflexible manifolds; a detailed discussion of these results is deferred to Appendix II.

\subsection{Strongly inflexible manifolds}

A manifold $M$ is inflexible if and only if the set $\operatorname{deg}(M, M)$ is finite. More ambitiously we can ask that $\operatorname{deg}(N, M)$ is finite for any oriented manifold $N$ of the same dimension as $M$. This leads to the notion of strongly inflexible manifolds: 
Definition 6.14 (Strongly inflexible manifold) We call an oriented closed connected $d$-dimensional manifold $M$ strongly inflexible if for any oriented closed connected $d$-dimensional manifold $N$ the $\operatorname{set} \operatorname{deg}(N, M)$ is finite.

Clearly, any strongly inflexible manifold is also inflexible.

Example 6.15 The simplicial volume can be used to show that oriented closed connected hyperbolic manifolds $M$ are strongly inflexible. If $N$ is an oriented closed connected manifold of dimension $\operatorname{dim} M$, then

$$
|\operatorname{deg} f| \leq \frac{\|N\|}{\|M\|}<\infty
$$

for any map $f: N \rightarrow M$; notice that $\|M\|>0$ as $M$ is hyperbolic.

Unfortunately, we do not know of any simply connected manifolds that are strongly inflexible. As in the case of inflexible manifolds, rational homotopy theory and the examples from Section 6.2 and Appendix I could be a good starting point for seeking strongly inflexible manifolds. However one sees that the necessary calculations, if they are possible, would be significantly more complicated than in the case of inflexible manifolds.

Question 6.16 Is every "random" Poincaré differential graded algebra of high formal dimension (strongly) inflexible?

A small piece of evidence supporting a positive answer to Question 6.16 is the bordism result in Proposition II.12.

\subsection{Flexible spaces and manifolds}

Clearly, all spheres (of nonzero dimension) are flexible manifolds, and products of oriented closed connected manifolds with flexible ones are flexible again. Further examples of flexible manifolds and spaces can be obtained via rational homotopy theory:

Proposition 6.17 (Simply connected manifolds of low dimension are flexible) Oriented closed simply connected formal manifolds are flexible. In particular, all oriented closed simply connected manifolds of dimension 6 or less are flexible.

Proof Formal oriented closed simply connected manifolds admit many self-maps of nontrivial degree [21] and so are flexible. Moreover, by a classical result in rational homotopy theory, all oriented closed simply connected manifolds of dimension at most 6 are formal [19, Proposition 4.6]. 
A natural generalisation of formality of minimal models is being pure:

Definition 6.18 (Pure) A Sullivan algebra $(\bigwedge V, d)$ is pure if $V$ is finite-dimensional and

$$
\left.d\right|_{V^{\text {even }}}=0 \quad \text { and } \quad d\left(V^{\text {odd }}\right) \subset \bigwedge V^{\text {even }} ;
$$

here, $V^{\text {even }}$ and $V^{\text {odd }}$ denote the even and the odd part respectively of the graded vector space $V$.

Proposition 6.19 (Pure rational spaces are "almost flexible") Let $X$ be a rational space whose minimal model is pure. Then every rational homology class of $X$ in positive degree is a sum of flexible homology classes.

Proof Let $A=(\bigwedge V, d)$ be the minimal model of $X$. In view of the equivalence of categories between the category of minimal Sullivan dgas (and homotopy classes of dga morphisms) and the category of rational spaces (and homotopy classes of continuous maps) it suffices to show that every cohomology class in $H^{*}(\bigwedge V, d) \cong H_{*}(X ; \mathbb{Q})$ in positive degree is a sum of flexible cohomology classes (as defined in Definition 6.3).

Let $f: \bigwedge V \rightarrow \bigwedge V$ be the algebra morphism uniquely determined by the maps

$$
\begin{aligned}
V^{\text {even }} \rightarrow V, & x \mapsto 2^{|x|} \cdot x, \\
V^{\text {odd }} \rightarrow V, & y \mapsto 2^{|y|-1} \cdot y .
\end{aligned}
$$

Using the fact that $(\bigwedge V, d)$ is pure, a straightforward computation shows that $f$ is compatible with $d$. On the even part, the differential vanishes, and so $\left.f \circ d\right|_{V^{\text {even }}}=$ $0=\left.d \circ f\right|_{V^{\text {even }}}$. The differential of an odd element $y \in V^{\text {odd }}$ of $V$ is a sum of products of even elements of $V$ whose degrees sum up to $|y|-1$, and so

$$
f \circ d(y)=2^{|y|-1} \cdot d y=d \circ f(y) .
$$

Because $A$ is pure, there is an additional grading on $A$ given by the word length in $V^{\text {odd }}$; more explicitly, $A=\bigoplus_{k \in \mathbb{N}} A_{[k]}$, where

$$
A_{[k]}:=\bigwedge V^{\text {even }} \otimes \bigwedge^{k} V^{\text {odd }}
$$

for all $k \in \mathbb{N}$ [7, page 435]; notice that the differential $d$ is homogeneous of degree -1 with respect to this grading and that $f(z)=2^{|z|-k} \cdot z$ holds for all $k \in \mathbb{N}$ and all $z \in A_{[k]}$. So the dga morphism $f$ witnesses that every cohomology class in $H^{*}(A)$ of nonzero degree that can be represented by a cocycle in one of the subspaces $A_{[k]}$ is flexible. On the other hand, using the direct sum decomposition $A=\bigoplus_{k \in \mathbb{N}} A_{[k]}$ and the fact that $d$ is homogeneous of degree -1 one can easily check that every cohomology class in $H^{*}(A)$ is a sum of cohomology classes represented by such cocycles. 
Flexibility as established in Propositions 6.17 and 6.19 provides a means to prove the vanishing of finite functorial seminorms on certain classes (Corollaries 7.7 and 7.8). Clearly, the same methods apply whenever the minimal models allow for an appropriate grading or weight function. For simplicity, we restricted ourselves to the cases above.

\section{Functorial seminorms on simply connected spaces}

In the following we discuss Gromov's question whether all functorial seminorms on singular homology are trivial on simply connected spaces (Question 1.1).

Here, a key role is played by simply connected inflexible manifolds. Recall that an oriented closed connected manifold $M$ is inflexible if

$$
\operatorname{deg}(M, M) \subset\{-1,0,1\} .
$$

We start with a construction of a functorial seminorm that is not trivial on all simply connected spaces (Section 7.1); on the other hand, we show in Section 7.2 that the finite case of Gromov's question can be answered affirmatively in all dimensions $d \leq 6$.

\subsection{Functorial seminorms that are nontrivial on certain simply connected spaces}

Using the construction from Section 4 and simply connected inflexible manifolds, we obtain a (possibly infinite) functorial seminorm that is nontrivial on simply connected spaces:

Recall that an oriented closed connected manifold $N$ is said to dominate an oriented closed connected manifold $M$ of the same dimension if there exists a continuous map $N \rightarrow M$ of nonzero degree.

Definition 7.1 (Domination $\mathrm{Mfd}_{d}$-seminorm associated with a $d$-manifold) Let $M$ be an oriented closed connected $d$-manifold. Then the domination $\mathrm{Mfd}_{d}$-seminorm $v_{M}: \operatorname{Mfd}_{d} \rightarrow[0, \infty]$ associated with $M$ is defined by

$$
\begin{aligned}
v_{M}(N): & =\sup \{|d| \mid d \in \operatorname{deg}(N, M)\} \\
& =\sup \{|\operatorname{deg} f| \mid f: N \rightarrow M \text { continuous }\} \in[0, \infty]
\end{aligned}
$$

for all $N \in \operatorname{Mfd}_{d}$.

Proposition 7.2 If $M$ is an oriented closed connected $d$-manifold, then the domination $\mathrm{Mfd}_{d}$-seminorm $v_{M}$ is functorial. 


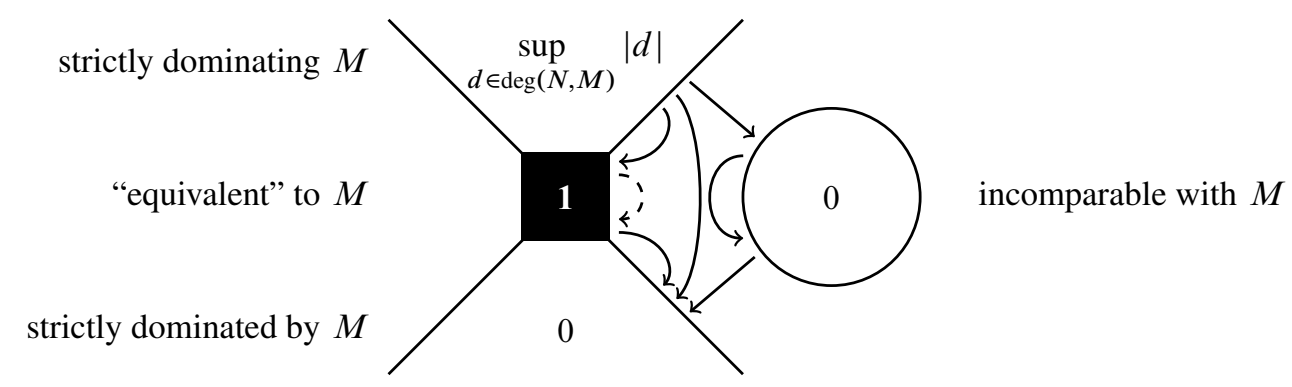

Figure 1: Schematic construction of $v_{M}$ in the proof of Corollary 7.4: the arrows indicate where maps of nonzero degree can exist, the dashed arrow indicates that only maps of degree $-1,0,1$ can exist.

Proof This follows from the definition of the domination seminorm and multiplicativity of the mapping degree.

Definition 7.3 (Domination seminorm associated with a $d$-manifold) Let $M$ be an oriented closed connected $d$-manifold. Then the domination seminorm $|\cdot|_{M}$ on singular homology of degree $d$ is the seminorm on singular homology in degree $d$ associated with $v_{M}$ (see Definition 4.1). By Proposition 7.2 and Theorem $4.2,|\cdot|_{M}$ is a functorial seminorm on $H_{d}(\cdot ; \mathbb{R})$.

Corollary 7.4 If $M$ is a simply connected closed inflexible manifold, then the domination seminorm $|\cdot|_{M}$ is not zero or infinite on all simply connected spaces. Hence there are functorial seminorms on singular homology that are not zero or infinite on all simply connected spaces.

Proof Let $M$ be a simply connected closed inflexible manifold; such a manifold exists by Theorem 6.8: we can even find such manifolds in infinitely many different dimensions (Corollary II.7). By Theorem 4.2(1) we have

$$
\left|[M]_{\mathbb{R}}\right|_{M}=v_{M}(M)=1 \notin\{0, \infty\} .
$$

Here $[M]_{\mathbb{R}}$ is of course the $\mathbb{R}$-fundamental class of the simply connected closed manifold $M$. We give a graphical description of the domination seminorm associated to $M$ in Figure 1.

Remark 7.5 We do not know whether the functorial seminorms constructed in Corollary 7.4 are finite. If $M$ is a an oriented closed connected $d$-manifold then by construction the domination $\mathrm{Mfd}_{d}$-seminorm $v_{M}$ is finite if and only if $M$ is strongly 
inflexible. It then follows by Theorem 4.2(2) and the definitions that the associated functorial seminorm $|\cdot|_{M}$ is finite if and only if $M$ is strongly inflexible. The existence of simply connected strongly inflexible manifolds remains open at the time of writing.

As we do not know of any simply connected strongly inflexible manifold, Gromov's question (Question 1.1(2)) remains open for finite functorial seminorms on singular homology.

\subsection{Partial results on finite functorial seminorms on simply connected spaces}

In view of the Hurewicz theorem, all finite functorial seminorms in degree 1, 2 or 3 vanish on simply connected spaces: any integral homology class of degree 1, 2 or 3 of a simply connected space can be represented by a sphere.

Proposition 7.6 For $d \in \mathbb{N}_{\geq 4}$ the following statements are equivalent.

(1) There is a finite functorial seminorm $|\cdot|$ on $H_{d}(\cdot ; \mathbb{R})$ such that for some homology class $\alpha \in H_{d}(X ; \mathbb{R})$ of some simply connected space $X$ we have $|\alpha| \neq 0$.

(2) There exists an oriented closed simply connected $d$-manifold that is strongly inflexible.

Proof First, let us assume that the first statement holds. Without loss of generality we may assume that $X$ is path-connected and (in view of the triangle inequality) that $\alpha$ is rational. By Corollary 3.2 we can write $\alpha=a \cdot H_{d}(f ; \mathbb{R})[M]_{\mathbb{R}}$, where $M$ is some oriented closed simply connected $d$-manifold, $f: M \rightarrow X$ is a continuous map and $a \in \mathbb{R} \backslash\{0\}$. We now show that the manifold $M$ is strongly inflexible: because $|\cdot|$ is finite and functorial, we obtain

$$
\infty>\left|[M]_{\mathbb{R}}\right| \geq \frac{1}{|a|} \cdot|\alpha|>0 .
$$

If $N$ is an oriented closed connected $d$-manifold, then for all continuous maps $g: N \rightarrow M$ it follows that

$$
|\operatorname{deg}(g)| \leq \frac{\left|[N]_{\mathbb{R}}\right|}{\left|[M]_{\mathbb{R}}\right|}<\infty .
$$

Hence, $\operatorname{deg}(N, M)$ is finite, and so $M$ is strongly inflexible.

Conversely, if there exists an oriented closed simply connected strongly inflexible $d-$ manifold $M$, we consider the functorial seminorm $|\cdot|$ associated with the domination seminorm $v_{M}$ for $M$ (see Section 7.1). Because $M$ is strongly inflexible, $v_{M}$ is finite. So by Theorem 4.2, also $|\cdot|$ is finite, and $\left|[M]_{\mathbb{R}}\right|=v_{M}(M)=1 \notin\{0, \infty\}$. 
Corollary 7.7 (Degrees 4, 5, and 6) All finite functorial seminorms in degree 4, 5 and 6 vanish on simply connected spaces.

Proof All oriented closed connected simply connected manifolds of dimension at most 6 are flexible (Proposition 6.17), and so cannot be strongly inflexible. Hence, the claim follows by applying Proposition 7.6.

Because finite functorial seminorms vanish on flexible homology classes, we obtain:

Corollary 7.8 Let $X$ be a rational space whose minimal model is pure. Then every finite functorial seminorm vanishes on every homology class of $X$ in positive degree.

Proof This is a direct consequence of the fact that rational spaces with pure minimal model are almost flexible (Proposition 6.19).

Moreover, it follows from Gaifullin's work [8] that finite functorial seminorms which are multiplicative with respect to finite coverings are trivial on simply connected spaces:

Definition 7.9 (URC-manifold [9, page 1747]) Let $d \in \mathbb{N}$. An oriented closed connected $d$-manifold $M$ is a URC-manifold (universal realisation of cycles), if for every topological space $X$ and every $\alpha \in H_{d}(X ; \mathbb{Z})$, there is a finite sheeted covering $\bar{M}$ of $M$, a map $f: \bar{M} \rightarrow X$, and $k \in \mathbb{Z} \backslash\{0\}$ such that

$$
H_{d}(f ; \mathbb{Z})([\bar{M}])=k \cdot \alpha \in H_{d}(X ; \mathbb{Z}) .
$$

Gaifullin proved that there are many URC-manifolds in each dimension [9, Theorem 1.3]. Clearly, any URC-manifold of dimension at least 2 is strongly inflexible and has nonzero simplicial volume, because its finite coverings dominate hyperbolic manifolds, which are strongly inflexible (Example 6.15).

Example 7.10 (Functorial seminorms associated with coverings of URC-manifolds) Let $d \in \mathbb{N}$, let $M$ be an oriented closed connected URC-manifold of dimension $d$ and let $S \subset \operatorname{Mfd}_{d}$ be the subclass of all finite connected covering spaces of $M$. Then $\left.v_{M}\right|_{S}$ is a functorial seminorm on $S$. If $d \geq 2$, then $M$ is strongly inflexible, and so $\left.v_{M}\right|_{S}$ is a finite functorial seminorm on $S$ with $\left.v_{M}\right|_{S}(M)=1$. More explicitly, multiplicativity under finite coverings and functoriality of simplicial volume show that

$$
\left.v_{M}\right|_{S}(N)=\frac{\|N\|}{\|M\|}=\text { number of sheets of any covering } N \rightarrow M
$$

holds for all $N \in S$.

Let $|\cdot|_{M}^{c}$ be the associated functorial seminorm on $H_{d}(\cdot ; \mathbb{R})$; because of the URCproperty, this functorial seminorm $|\cdot|_{M}^{c}$ is finite. 
Proposition 7.11 (Multiplicative finite functorial seminorms) Let $d \in \mathbb{N}$ and let $|\cdot|$ be a finite functorial seminorm on $H_{d}(\cdot ; \mathbb{R})$ that is multiplicative with respect to finite coverings, ie satisfying: for all topological spaces $X$, all finite coverings $p: Y \rightarrow X$ and all $\alpha \in H_{d}(Y ; \mathbb{R})$ we have

$$
\left|H_{d}(p ; \mathbb{R})(\alpha)\right|=\frac{1}{k} \cdot|\alpha|,
$$

where $k$ denotes the number of sheets of $p$. Then there exists a constant $c \in \mathbb{R}_{\geq 0}$ such that for all topological spaces $X$ and all $\alpha \in H_{d}(X ; \mathbb{R})$ we have

$$
|\alpha| \leq c \cdot\|\alpha\|_{1} .
$$

In particular, $|\cdot|$ is trivial on simply connected spaces.

Proof Without loss of generality we may assume that $d \geq 2$. Let $M$ be an oriented closed connected URC-manifold $M$ in dimension $d$. It follows from the arguments of Gaifullin that there is a constant $a \in \mathbb{R}_{\geq 0}$ satisfying (see [8, Proposition 6.2])

$$
|\cdot|_{M}^{c} \leq a \cdot\|\cdot\|_{1} \cdot
$$

On the other hand, multiplicativity of $|\cdot|$ and the construction of $|\cdot|_{M}^{c}$ (Example 7.10) show that

$$
\left|[N]_{\mathbb{R}}\right|=\left|[M]_{\mathbb{R}}\right| \cdot\left|[N]_{\mathbb{R}}\right|_{M}^{c}
$$

holds for all $N \in S$ and hence that $|\cdot| \leq\left|[M]_{\mathbb{R}}\right| \cdot|\cdot|{ }_{M}^{c}$. Therefore,

$$
|\cdot| \leq a \cdot\left|[M]_{\mathbb{R}}\right| \cdot\|\cdot\|_{1} .
$$

\section{Appendix I: Four inflexible Poincaré dgas}

This appendix is devoted to the algebraic side of inflexibility: we construct the four inflexible Poincaré differential graded algebras used in Section 6. We explain the construction in Section I.1. In Section I.2, we prove that these dgas are Poincare dgas; the intersection forms are computed in Section I.3. In Section I.4, we show that these dgas are inflexible.

\section{I.1 A design pattern for possibly inflexible dgas}

We start by defining a collection of dgas; all of the four concrete examples below follow the same design pattern based on two examples of Arkowitz and Lupton [2, Example 5.1 and 5.2], which are respectively examples $A_{3}$ and $A_{4}$ below. We shall construct dgas having the following properties: 
- Two generators $x_{1}, x_{2}$ of even degree with trivial differential.

- Four generators $y_{1}, y_{2}, y_{3}, z$ of odd degree; the differential is given by

$$
d y_{1}:=x_{1}^{3} x_{2}, \quad d y_{2}:=x_{1}^{2} x_{2}^{2}, \quad d y_{3}:=x_{1} x_{2}^{3},
$$

and for the differential of $z$ we choose $z^{\prime} \in \bigwedge\left(x_{1}, x_{2}, y_{1}, y_{2}, y_{4}\right)$ in such a way that $d\left(y_{1} y_{2} y_{3}\right)=x_{1}^{k} \cdot z^{\prime}$ or $d\left(y_{1} y_{2} y_{3}\right)=x_{2}^{k} \cdot z^{\prime}$ and set

$$
d z:=z^{\prime}+x_{1}^{k_{1}}+x_{2}^{k_{2}}
$$

with suitable exponents $k, k_{1}, k_{2} \in \mathbb{N}_{>0}$.

By construction, $d \circ d\left(y_{j}\right)=0$ for all $j \in\{1,2,3\}$ and $d \circ d(z)=0$; moreover, these dgas are finitely generated minimal dgas.

The following four example dgas

$$
A_{j}:=\left(\bigwedge\left(x_{1}, x_{2}, y_{1}, y_{2}, y_{3}, z\right), d\right)
$$

with $j \in\{1, \ldots, 4\}$ are all of this kind:

Example I.1 ( $A_{1}$ : an elliptic inflexible dga of formal dimension 64) Define the dga $A_{1}$ with generators of degrees

$$
\left(\left|x_{1}\right|,\left|x_{2}\right|,\left|y_{1}\right|,\left|y_{2}\right|,\left|y_{3}\right|,|z|\right)=(2,4,9,11,13,35),
$$

where the differential $d$ is given by

$$
\begin{aligned}
d x_{1} & :=0, \quad d y_{1}:=x_{1}^{3} x_{2}, & d z:=x_{2}^{4} y_{1} y_{2}-x_{1} x_{2}^{3} y_{1} y_{3}+x_{1}^{2} x_{2}^{2} y_{2} y_{3}+x_{1}^{18}+x_{2}^{9} \\
d x_{2}:=0, \quad d y_{2} & :=x_{1}^{2} x_{2}^{2}, & =x_{2}^{2} \cdot w+x_{1}^{18}+x_{2}^{9}, \\
& d y_{3}:=x_{1} x_{2}^{3}, &
\end{aligned}
$$

where we use the abbreviation $w:=x_{2}^{2} y_{1} y_{2}-x_{1} x_{2} y_{1} y_{3}+x_{1}^{2} y_{2} y_{3}$; in other words $x_{1} x_{2} w=d\left(y_{1} y_{2} y_{3}\right)$.

Example I.2 ( $A_{2}$ : An elliptic inflexible dga of formal dimension 108) Define the dga $A_{2}$ with generators of degrees

$$
\left(\left|x_{1}\right|,\left|x_{2}\right|,\left|y_{1}\right|,\left|y_{2}\right|,\left|y_{3}\right|,|z|\right)=(4,6,17,19,21,59),
$$

where the differential $d$ is given by

$$
\begin{aligned}
d x_{1} & :=0, \quad d y_{1}:=x_{1}^{3} x_{2}, \quad d z:=x_{2}^{4} y_{1} y_{2}-x_{1} x_{2}^{3} y_{1} y_{3}+x_{1}^{2} x_{2}^{2} y_{2} y_{3}+x_{1}^{15}+x_{2}^{10} . \\
d x_{2}:=0, \quad d y_{2}: & =x_{1}^{2} x_{2}^{2}, \\
d y_{3} & :=x_{1} x_{2}^{3},
\end{aligned}
$$


Example I.3 $\left(A_{3}:\right.$ An elliptic inflexible dga of formal dimension 208 [2, Example 5.1]) Define the dga $A_{3}$ with generators of degrees

$$
\left(\left|x_{1}\right|,\left|x_{2}\right|,\left|y_{1}\right|,\left|y_{2}\right|,\left|y_{3}\right|,|z|\right)=(8,10,33,35,37,119),
$$

where the differential $d$ is given by

$$
\begin{aligned}
d x_{1}:=0, & d y_{1}:=x_{1}^{3} x_{2}, \quad d z:=x_{1}^{4} x_{2}^{2} y_{1} y_{2}-x_{1}^{5} y_{1} y_{3}+x_{1}^{6} y_{2} y_{3}+x_{1}^{15}+x_{2}^{12} . \\
d x_{2}:=0, \quad d y_{2} & :=x_{1}^{2} x_{2}^{2}, \\
d y_{3} & :=x_{1} x_{2}^{3},
\end{aligned}
$$

Example I.4 ( $A_{4}$ : An elliptic inflexible dga of formal dimension 228 [2, Example 5.2]) Define the dga $A_{4}$ with generators of degrees

$$
\left(\left|x_{1}\right|,\left|x_{2}\right|,\left|y_{1}\right|,\left|y_{2}\right|,\left|y_{3}\right|,|z|\right)=(10,12,41,43,45,119),
$$

where the differential $d$ is given by

$$
\begin{aligned}
d x_{1} & :=0, \quad d y_{1}:=x_{1}^{3} x_{2}, \quad d z:=x_{2}^{3} y_{1} y_{2}-x_{1} x_{2}^{2} y_{1} y_{3}+x_{1}^{2} x_{2} y_{2} y_{3}+x_{1}^{12}+x_{2}^{10} . \\
d x_{2} & :=0, \quad d y_{2}:=x_{1}^{2} x_{2}^{2}, \\
d y_{3}: & :=x_{1} x_{2}^{3},
\end{aligned}
$$

We will carry out the proofs in detail only for the dga $A_{1}$ defined in Example I.1; in fact, this is the most complicated of the four examples and the other examples can be treated by analogous arguments and calculations.

\section{I.2 The example dgas are Poincaré dgas}

Recall that a minimal Sullivan algebra $(\bigwedge V, d)$ is called elliptic if $V$ and $H^{*}(\bigwedge V, d)$ are finite dimensional.

Proposition I.5 (Ellipticity) The dgas $A_{1}, A_{2}, A_{3}$ and $A_{4}$ are elliptic.

Proof As these dgas are finitely generated by construction, it suffices to show that their cohomology is finite-dimensional. In other words, it suffices to show that the cohomology is generated by nilpotent classes. Because the odd degree generators are nilpotent on the level of the dgas and because the differential is trivial on the even degree generators, it suffices to show that the classes $\left[x_{1}\right]$ and $\left[x_{2}\right]$ are nilpotent.

We now show that $\left[x_{1}\right]$ and $\left[x_{2}\right]$ are nilpotent in $H^{*}\left(A_{1}\right)$ (the arguments for the other example dgas are similar). By definition of $d$, we have

$\left[x_{1}\right]^{19}=\left[x_{1}^{19}\right]=\left[x_{1} d z-x_{2} d\left(y_{1} y_{2} y_{3}\right)-x_{1} x_{2}^{9}\right]=\left[d\left(x_{1} z-x_{2} y_{1} y_{2} y_{3}-x_{2} y_{3}\right)\right]=0$. 
Therefore we obtain

$$
\begin{aligned}
{\left[x_{2}\right]^{18} } & =\left[x_{2}^{9}\right] \cdot\left[x_{2}^{9}\right]=\left(\left[d z-x_{2}^{2} w-x_{1}^{18}\right]\right)^{2}=\left(\left[x_{2}^{2} w\right]-\left[x_{1}^{18}\right]\right)^{2} \\
& =\left[\left(x_{2}^{2} w\right)^{2}\right]-2\left[x_{1}^{18} x_{2}^{2} w\right]+\left[x_{1}\right]^{36}=0-2\left[d\left(x_{1}^{17} x_{2} y_{1} y_{2} y_{3}\right)\right]+0=0 ;
\end{aligned}
$$

notice that $w^{2}=0$ because every summand of $w$ contains two of the three odd generators $y_{1}, y_{2}, y_{3}$ and $y_{j}^{2}=0$.

We will now select nonzero classes in the top cohomology, which will play the role of fundamental classes:

Proposition I.6 (Fundamental classes for $A_{1}, A_{2}, A_{3}, A_{4}$ )

(1) The class $\left[x_{2}\right]^{16}$ is nonzero in $H^{64}\left(A_{1}\right)$.

(2) The class $\left[x_{2}\right]^{18}$ is nonzero in $H^{108}\left(A_{2}\right)$.

(3) The class $\left[x_{1}\right]^{26}$ is nonzero in $H^{208}\left(A_{3}\right)$.

(4) The class $\left[x_{2}\right]^{19}$ is nonzero in $H^{228}\left(A_{4}\right)$.

Proof We give the proof only for $A_{1}$, the other cases being similar. Assume for a contradiction that $\left[x_{2}^{16}\right]=0$ in $H^{64}\left(A_{1}\right)$; hence, there is an element $u$ of $A_{1}$ of degree 63 with $d u=x_{2}^{16}$. We can write $u$ as

$$
u=p z+p_{12} y_{1} y_{2} z+p_{13} y_{1} y_{3} z+p_{23} y_{2} y_{3} z+p_{1} y_{1}+p_{2} y_{2}+p_{3} y_{3},
$$

where $p, p_{12}, p_{13}, p_{23}, p_{1}, p_{2}, p_{3}$ are homogeneous polynomials in $x_{1}, x_{2}$. Then $x_{2}^{16}=d u=p x_{2}^{9}+p x_{1}^{18}+p x_{2}^{4} y_{1} y_{2}-p x_{1} x_{2}^{3} y_{1} y_{3}+p x_{1}^{2} x_{2}^{2} y_{2} y_{3}$

$$
\begin{aligned}
& +p_{12} d\left(y_{1} y_{2}\right) z+p_{12} x_{2}^{9} y_{1} y_{2}+p_{12} x_{1}^{18} y_{1} y_{2}+0 \\
& +p_{13} d\left(y_{1} y_{3}\right) z+p_{13} x_{2}^{9} y_{1} y_{3}+p_{13} x_{1}^{18} y_{1} y_{3}+0 \\
& +p_{23} d\left(y_{2} y_{3}\right) z+p_{23} x_{2}^{9} y_{2} y_{3}+p_{23} x_{1}^{18} y_{2} y_{3}+0+q,
\end{aligned}
$$

where $q$ is a homogeneous polynomial in $x_{1}, x_{2}$ that is divisible by $x_{1} x_{2}$; the zeroes at the end of the lines stem from the fact that squares of odd degree elements are zero and each summand of $w$ contains two of the three odd degree generators $y_{1}, y_{2}, y_{3}$.

Because $A_{1}$ is freely generated by $x_{1}, \ldots, z$, comparing the $x_{2}^{16}$-coefficients on both sides shows that $p \neq 0$. Moreover, comparing the $z$-coefficients of both sides yields

$$
p_{12} d\left(y_{1} y_{2}\right)+p_{13} d\left(y_{1} y_{3}\right)+p_{23} d\left(y_{2} y_{3}\right)=0 \text {. }
$$

Comparing the coefficients of $y_{1}, y_{2}, y_{3}$ in this equation gives us

$$
-x_{1}^{2} x_{2}^{2} p_{12}=x_{1} x_{2}^{3} p_{13}, \quad x_{1}^{3} x_{2} p_{12}=x_{1} x_{2}^{3} p_{23}, \quad x_{1}^{3} x_{2} p_{13}=-x_{1}^{2} x_{2}^{2} p_{23} .
$$


Because $\operatorname{deg} p_{12}=8, \operatorname{deg} p_{13}=6$ and $\operatorname{deg} p_{23}=4$, a simple divisibility argument shows that there is an $\eta \in \mathbb{Q}$ with

$$
p_{13}=-\eta \cdot x_{1} x_{2}, \quad p_{23}=\eta \cdot x_{1}^{2}, \quad p_{12}=\eta \cdot x_{2}^{2} .
$$

Hence, comparing the summands of $d u$ that are divisible by $y_{1} y_{2}$, but not by $z$, shows

$$
0=p x_{2}^{4}+p_{12} x_{2}^{9}+p_{12} x_{1}^{18}=p x_{2}^{4}+\eta \cdot x_{2}^{11}+\eta \cdot x_{1}^{18} x_{2}^{2} .
$$

Because $p \neq 0$, it follows that $\eta=0$ (otherwise the last summand is not divisible by $x_{2}^{4}$ ). On the other hand, by an analogous argument, we obtain

$$
0=-p x_{1} x_{2}^{3}+p_{13} x_{2}^{9}+p_{13} x_{1}^{18}=-p x_{1} x_{2}^{3}-\eta \cdot x_{1} x_{2}^{10}-\eta \cdot x_{1}^{19} x_{2},
$$

and thus $p=0$, contradicting $p \neq 0$. So $x_{2}^{16}$ cannot be a coboundary.

Corollary I.7 (The dgas $A_{1}, \ldots, A_{4}$ are Poincaré dgas) The dgas $A_{1}, \ldots, A_{4}$ are Poincare dgas with the cohomology classes in Proposition I.6 as fundamental classes.

Proof The dgas $A_{1}, \ldots, A_{4}$ are elliptic (Proposition I.5). By a classical result in rational homotopy theory [7, Proposition 38.3], cohomology algebras of elliptic minimal Sullivan algebras are Poincaré duality algebras; clearly, any nonzero cohomology class in the top cohomology can be chosen as fundamental class.

\section{I.3 The intersection forms of the example dgas}

Proposition I.8 (Intersection form of $A_{1}$ ) The classes $\left[x_{2} w\right],\left[x_{1}^{2} w\right],\left[x_{1}^{16}\right]$ and $\left[x_{2}^{8}\right]$ form a $\mathbb{Q}$-basis of $H^{32}\left(A_{1}\right)$ (the middle cohomology of $A_{1}$ ), and the intersection form with respect to this basis and the fundamental class $\left[x_{2}^{16}\right]$ of $A_{1}$ (see Proposition I.6) looks as follows:

$$
\left(\begin{array}{rrrr}
0^{(3)} & 0^{(3)} & 0^{(6)} & -1^{(7)} \\
0^{(3)} & 0^{(3)} & 1^{(4)} & 0^{(5)} \\
0^{(6)} & 1^{(4)} & 0^{(2)} & 0^{(1)} \\
-1^{(7)} & 0^{(5)} & 0^{(1)} & 1^{(0)}
\end{array}\right)
$$

(The superscripts in the matrix refer to the part of the proof where the corresponding coefficient is computed.)

Proof We first show that $H^{32}\left(A_{1}\right)$ is generated by $\left[x_{2} w\right],\left[x_{1}^{2} w\right],\left[x_{1}^{16}\right]$ and $\left[x_{2}^{8}\right]$. What do cocycles of degree 32 in $A_{1}$ look like? Clearly, $x_{1}^{16}$ and $x_{2}^{8}$ are cocycles of degree 32. All cocycles in the subalgebra $\bigwedge\left(x_{1}, x_{2}\right)$ divisible by $x_{1} x_{2}$ are in 
the image of $d$ (by definition of $d y_{1}, d y_{2}, d y_{3}$ ). Because the differential is trivial on $\bigwedge\left(x_{1}, x_{2}\right)$, it remains to look at cocycles of the form

$$
u=p_{12} y_{1} y_{2}+p_{13} y_{1} y_{3}+p_{23} y_{2} y_{3},
$$

where $p_{12}, p_{13}, p_{23} \in \bigwedge\left(x_{1}, x_{2}\right)$. If $d u=0$, then looking the coefficients of $y_{1}, y_{2}$ and $y_{3}$ respectively in $d u$ leads to

$$
x_{1}^{3} x_{2} p_{12}=x_{1} x_{2}^{3} p_{23}, \quad x_{1}^{2} x_{2}^{2} p_{12}=-x_{1} x_{2}^{3} p_{13}, \quad x_{1}^{3} x_{2} p_{13}=-x_{1}^{2} x_{2}^{2} p_{23} .
$$

As $\operatorname{deg} p_{12}=12, \operatorname{deg} p_{13}=10$, and $\operatorname{deg} p_{23}=8$, a simple divisibility consideration shows that there exist $\eta_{1}, \eta_{2} \in \mathbb{Q}$ such that

$p_{23}=\eta_{1} \cdot x_{1}^{4}+\eta_{2} \cdot x_{1}^{2} x_{2}, \quad p_{12}=\eta_{1} \cdot x_{1}^{2} x_{2}^{2}+\eta_{2} \cdot x_{2}^{3}, \quad p_{13}=-\eta_{1} \cdot x_{1}^{3} x_{2}-\eta_{2} \cdot x_{1} x_{2}^{2}$;

hence, $u=\eta_{1} \cdot x_{1}^{2} w+\eta_{2} \cdot x_{2} w$. So $H^{32}\left(A_{1}\right)$ indeed is generated as $\mathbb{Q}$-vector space by $\left[x_{2} w\right],\left[x_{1}^{2} w\right],\left[x_{1}^{16}\right]$ and $\left[x_{2}^{8}\right]$.

As next step, we determine the corresponding matrix for the intersection form with respect to the fundamental class $\left[x_{2}^{16}\right]$.

(0) Because we chose $\left[x_{2}^{16}\right]$ as fundamental class with respect to which the intersection form is computed, the matrix coefficient corresponding to column $\left[x_{2}^{8}\right]$ and row $\left[x_{2}^{8}\right]$ equals 1 .

(1) We have $\left[x_{1}^{16}\right] \cdot\left[x_{2}^{8}\right]=\left[d\left(x_{1}^{13} x_{2}^{7} y_{1}\right)\right]=0$.

(2) Moreover, $\left[x_{1}^{16}\right] \cdot\left[x_{1}^{16}\right]=\left[x_{1}^{32}\right]=0$ as was shown in the proof of Proposition I.5.

(3) Squares of elements of $A_{1}$ of odd degree are zero, and hence $w^{2}=0$ (because each summand of $w$ contains two of the three odd elements $\left.y_{1}, y_{2}, y_{3}\right)$.

(4) We have (because $(d z) w=0=w w$ as in the previous item)

$$
\begin{aligned}
{\left[x_{1}^{16}\right] \cdot\left[x_{1}^{2} w\right] } & =\left[(d z) \cdot w-x_{2}^{9} w-x_{2}^{2} w w\right]=\left[0-x_{2}^{9} w-0\right] \\
& =-\left[d\left(x_{2}^{7} z\right)+x_{2}^{16}+x_{2}^{7} x_{1}^{18}\right]=-\left[x_{2}^{16}\right]+\left[d\left(x_{1}^{15} x_{2}^{6} y_{1}\right)\right]=-\left[x_{2}^{16}\right] .
\end{aligned}
$$

(5) Moreover, $\left[x_{2}^{8}\right] \cdot\left[x_{1}^{2} w\right]=\left[d\left(x_{2}^{7} x_{1} y_{1} y_{2} y_{3}\right)\right]=0$.

(6) Analogously, $\left[x_{1}^{16}\right] \cdot\left[x_{2} w\right]=\left[d\left(x_{1}^{15} y_{1} y_{2} y_{3}\right)\right]=0$.

(7) Finally,

$$
\left[x_{2}^{8}\right] \cdot\left[x_{2} w\right]=\left[d\left(x_{2}^{7} z\right)-x_{1}^{18} x_{2}^{7}-x_{2}^{16}\right]=\left[-d\left(x_{1}^{15} x_{2}^{6} y_{1}\right)-x_{2}^{16}\right]=-\left[x_{2}^{16}\right] .
$$

Moreover, from the shape of this matrix we can easily deduce that the elements $\left[x_{2} w\right]$, $\left[x_{1}^{2} w\right],\left[x_{1}^{16}\right]$ and $\left[x_{2}^{8}\right]$ are linearly independent over $\mathbb{Q}$. 
Remark I.9 (Intersection form of $A_{2}, A_{3}, A_{4}$ ) Similarly to the previous proposition one can show the following.

- The classes $\left[x_{2}^{3} y_{1} y_{2}-x_{1} x_{2}^{2} y_{1} y_{3}+x_{1}^{2} x_{2} y_{2} y_{3}\right],\left[x_{2}\right]^{8}$ form a $\mathbb{Q}$-basis of the middle cohomology $H^{54}\left(A_{2}\right)$ of $A_{2}$. The intersection form of $A_{2}$ with respect to this basis and the fundamental class $\left[x_{2}\right]^{18}$ of $A_{2}$ is

$$
\left(\begin{array}{rr}
0 & -1 \\
-1 & 1
\end{array}\right)
$$

- The classes $\left[x_{1}^{2} x_{2}^{2} y_{1} y_{2}-x_{1}^{3} x_{2} y_{1} y_{3}+x_{1}^{4} y_{2} y_{3}\right],\left[x_{1}\right]^{13}$ form a $\mathbb{Q}$-basis of the middle cohomology $H^{104}\left(A_{3}\right)$ of $A_{3}$. The intersection form of $A_{3}$ with respect to this basis and the fundamental class $\left[x_{1}\right]^{26}$ is

$$
\left(\begin{array}{rr}
0 & -1 \\
-1 & 1
\end{array}\right)
$$

- The middle cohomology $H^{114}\left(A_{4}\right)$ of $A_{4}$ is zero.

\section{I.4 The example dgas are inflexible}

We now show that the four Poincaré dgas $A_{1}, A_{2}, A_{3}$ and $A_{4}$ are inflexible in the sense that there is no dga morphism whose induced homomorphism on cohomology maps the fundamental class to a nontrivial multiple of itself.

Proposition I.10 (Inflexibility) The dgas $A_{1}, A_{2}, A_{3}$, and $A_{4}$ are inflexible.

Proof We will give the complete calculation only for the example $A_{1}$; for the other dgas the calculation is similar, and even a bit simpler as the degrees of the even generators $x_{1}$ and $x_{2}$ are less entangled; moreover, for the dgas $A_{3}$ and $A_{4}$ an argument is contained in the work of Arkowitz and Lupton [2, Examples 5.1 and 5.2]. Let $f: A_{1} \rightarrow A_{1}$ be a dga morphism; looking at the degrees of the generators of $A_{1}$ we see that there are constants $\alpha_{1}, \alpha_{2}, \alpha_{2,1}, \ldots, \gamma, \gamma_{1} \in \mathbb{Q}$ and homogenous polynomials $p_{1}, p_{2}, p_{3}$ in $x_{1}, x_{2}$ such that

$$
\begin{aligned}
f\left(x_{1}\right) & =\alpha_{1} \cdot x_{1}, \\
f\left(x_{2}\right) & =\alpha_{2} \cdot x_{2}+\alpha_{2,1} \cdot x_{1}^{2}, \\
f\left(y_{1}\right) & =\beta_{1} \cdot y_{1}, \\
f\left(y_{2}\right) & =\beta_{2} \cdot y_{2}+\beta_{2,1} \cdot x_{1} y_{1}, \\
f\left(y_{3}\right) & =\beta_{3} \cdot y_{3}+\beta_{3,1} \cdot x_{1}^{2} y_{1}+\beta_{3,2} \cdot x_{2} y_{1}+\beta_{3,3} \cdot x_{1} y_{2}, \\
f(z) & =\gamma \cdot z+\gamma_{1} \cdot x_{1} y_{1} y_{2} y_{3}+p_{1} y_{2}+p_{2} y_{2}+p_{3} y_{3} .
\end{aligned}
$$


Using that $f$ as a dga morphism is compatible with the differential $d$ of $A_{1}$ and that $A_{1}$ is freely generated by $x_{1}, \ldots, z$, we deduce constraints on the coefficients $\alpha_{1}, \ldots$. Notice that because we chose $\left[x_{2}^{16}\right]$ as fundamental class of $A_{1}$, we can read off the "degree" of $f$ from the coefficient $\alpha_{2}$, and it suffices to show that $\alpha_{2} \in\{-1,0,1\}$.

(1) Comparing the coefficients for $f \circ d\left(y_{1}\right)$ and $d \circ f\left(y_{1}\right)$, we obtain

$$
\beta_{1}=\alpha_{1}^{3} \alpha_{2}
$$

and $\alpha_{1}^{3} \alpha_{2,1}=0$. In particular, $\alpha_{1}=0$ or $\alpha_{2,1}=0$.

(2) Comparing the coefficients for $f \circ d\left(y_{2}\right)$ and $d \circ f\left(y_{2}\right)$, we additionally have

$$
\beta_{2}=\alpha_{1}^{2} \alpha_{2}^{2} \text {. }
$$

(3) Moreover, we have

$$
\begin{aligned}
& f \circ d(z)=\alpha_{1}^{18} \cdot x_{1}^{18}+\left(\alpha_{2} \cdot x_{2}+\alpha_{2,1} \cdot x_{1}^{2}\right)^{9}+\alpha_{2}^{4} \beta_{1} \beta_{2} \cdot x_{2}^{4} y_{1} y_{2}+q, \\
& d \circ f(z)=\gamma \cdot x_{1}^{18}+\gamma \cdot x_{2}^{9}+\gamma \cdot d\left(x_{2} w\right)+\gamma_{1} \cdot d\left(x_{1} y_{1} y_{2} y_{3}\right),
\end{aligned}
$$

where $q \in\left(x_{1} x_{2}\right) \cdot A_{1}$. Comparing the coefficients of these elements shows that

$$
\alpha_{1}^{18}+\alpha_{2,1}^{9}=\gamma=\alpha_{2}^{9} \text {. }
$$

Because $\gamma \cdot d\left(x_{2} w\right)+\gamma_{1} \cdot d\left(x_{1} y_{1} y_{2} y_{3}\right)$ and $q$ are divisible by $x_{1} x_{2}$,

$$
\gamma=\alpha_{2}^{4} \beta_{1} \beta_{2}=\alpha_{2}^{7} \alpha_{1}^{5}
$$

(in the second equation we used the results from steps (1) and (2)).

In view of step (1) we can assume that $\alpha_{1}=0$ or $\alpha_{2,1}=0$. If $\alpha_{1}=0$, then also $\alpha_{2}^{9}=\gamma=\alpha_{2}^{7} \alpha_{1}^{5}=0$ by step (3). On the other hand, if $\alpha_{1} \neq 0$ and $\alpha_{2,1}=0$, then

$$
\alpha_{1}^{18}=\gamma=\alpha_{2}^{9} \quad \text { and } \quad \alpha_{2}^{7} \alpha_{1}^{5}=\gamma=\alpha_{2}^{9}
$$

by step (3). Now a small computation shows that $\alpha_{2}=1$. Hence, $A_{1}$ is inflexible.

\section{Appendix II: More inflexible dgas and manifolds}

In this appendix we produce more examples of inflexible manifolds from the basic examples of Section 6 and Appendix I. Using connected sums and products, we obtain in infinitely many dimensions infinitely many homotopy types of oriented closed simply connected inflexible manifolds (Sections II.1 and II.2). Moreover, we show that inflexibility is "generic" in the sense that in infinitely many dimensions, every oriented 
bordism class can be rationally represented by a simply connected inflexible manifold (Section II.3).

Recall that if $(A,[A])$ is a Poincaré dga (Definition 6.4) then $\mathcal{M}(A,[A])$ denotes the class of all oriented closed simply connected manifolds that have trivial total Pontryagin class and realise this rational data (Definition 6.7).

\section{II.1 Inflexible connected sums}

In general, it is not clear that connected sums of inflexible manifolds are inflexible; however, in certain cases inflexibility is preserved under connected sums:

Theorem II.1 (Inflexible connected sums) Let $M$ be an oriented closed simply connected $n$-manifold with inflexible minimal model and $\pi_{n-1}(M) \otimes \mathbb{Q}=0$. Suppose that $N_{1}, \ldots, N_{r}$ are oriented closed simply connected $n$-manifolds such that $\operatorname{deg}\left(N_{j, \mathbb{Q}}, M_{\mathbb{Q}}\right)$ is finite for each $j \in\{1, \ldots, r\}$. Then the iterated connected sum

$$
M \# N_{1} \# \cdots \# N_{r}
$$

is inflexible. In particular, for all $r \in \mathbb{N}$ the $r$-fold connected sum $M^{\# r}$ is inflexible.

The proof of this theorem relies on applying repeatedly the following lemma. Recall that $\operatorname{deg}(N, M)$ is the set of degrees of maps between oriented closed connected manifolds $N$ and $M$; also, for subsets $A, B \subset \mathbb{Z}$ we write

$$
A+B:=\{a+b \mid a \in A, b \in B\} \subset \mathbb{Z} .
$$

Lemma II.2 Let $N_{1}, N_{2}$ and $M$ be oriented closed simply connected $n$-manifolds with rationalisations $N_{1, \mathbb{Q}}, N_{2, \mathbb{Q}}$, and $M_{\mathbb{Q}}$. If $\pi_{n-1}\left(M_{\mathbb{Q}}\right)=0$ then

$$
\operatorname{deg}\left(N_{1} \# N_{2}, M\right) \subset \operatorname{deg}\left(N_{1, \mathbb{Q}}, M_{\mathbb{Q}}\right)+\operatorname{deg}\left(N_{2, \mathbb{Q}}, M_{\mathbb{Q}}\right) .
$$

Proof Because rationalisation preserves rational cohomology, we have

$$
\operatorname{deg}\left(N_{1} \# N_{2}, M\right) \subset \operatorname{deg}\left(\left(N_{1} \# N_{2}\right) \mathbb{Q}, M_{\mathbb{Q}}\right)
$$

so it suffices to show $\operatorname{deg}\left(\left(N_{1} \# N_{2}\right)_{\mathbb{Q}}, M_{\mathbb{Q}}\right) \subset \operatorname{deg}\left(N_{1, \mathbb{Q}}, M_{\mathbb{Q}}\right)+\operatorname{deg}\left(N_{2, \mathbb{Q}}, M_{\mathbb{Q}}\right)$. To this end, we consider the cofibration sequence

$$
S^{n-1} \rightarrow N_{1} \# N_{2} \rightarrow\left(N_{1} \# N_{2}\right) \cup_{S^{n-1}} D^{n},
$$

where we attach $D^{n}$ along the inclusion $i: S^{n-1} \rightarrow N_{1} \# N_{2}$, where $S^{n-1}$ is the locus of the connected sum between $N_{1}$ and $N_{2}$. Clearly, the space $W:=\left(\left(N_{1} \# N_{2}\right) \cup_{S^{n-1}} D^{n}\right)$ is homotopic to the wedge $N_{1} \vee N_{2}$ : we will use this fact below. From the cofibration 
sequence (1) and its rationalisation we obtain the following commutative diagram of exact sequences:

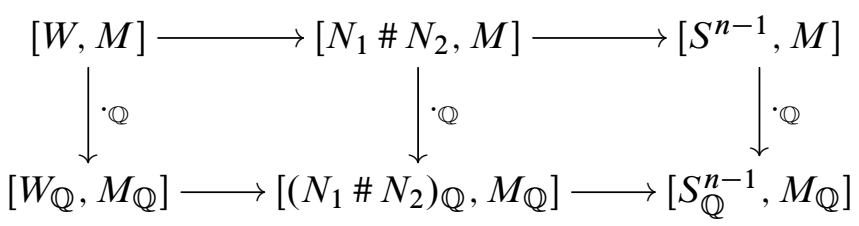

The lower sequence can be seen to be exact by looking at a concrete description of Sullivan models of cell additions (up to quasi-isomorphism) [7, Diagram 13.15].

But $\left[S_{\mathbb{Q}}^{n-1}, M_{\mathbb{Q}}\right] \cong \pi_{n-1}\left(M_{\mathbb{Q}}\right)=0$ by assumption. Thus, up to homotopy every map from the connected sum $\left(N_{1} \# N_{2}\right)_{\mathbb{Q}} \rightarrow M_{\mathbb{Q}}$ factors through the map $\left(N_{1} \# N_{2}\right)_{\mathbb{Q}} \rightarrow W_{\mathbb{Q}}$ induced by the inclusion. We observed above that there is a homotopy equivalence $W \simeq N_{1} \vee N_{2}$. The characterisation of rationalisations in terms of singular homology with integral coefficients [7, Theorems 9.3 and 9.6] together with the Mayer-Vietoris sequence in homology show that $W_{\mathbb{Q}} \simeq N_{1, \mathbb{Q}} \vee N_{2, \mathbb{Q}}$. Hence we have the equality

$$
\left[W_{\mathbb{Q}}, M_{\mathbb{Q}}\right]=\left[N_{1, \mathbb{Q}}, M_{\mathbb{Q}}\right] \times\left[N_{2, \mathbb{Q}}, M_{\mathbb{Q}}\right]
$$

So from the commutative diagram (2) above we see that there is an inclusion $\operatorname{deg}\left(\left(N_{1} \#\right.\right.$ $\left.\left.\left.N_{2}\right)_{\mathbb{Q}}, M_{\mathbb{Q}}\right)\right) \subset \operatorname{deg}\left(N_{1, \mathbb{Q}}, M_{\mathbb{Q}}\right)+\operatorname{deg}\left(N_{2, \mathbb{Q}}, M_{\mathbb{Q}}\right)$, as desired.

Proof of of Theorem II.1 For $N:=M \# N_{1} \# \ldots \# N_{r}$, observe that the obvious collapse map $N \rightarrow M$ has degree 1 and so $1 \in \operatorname{deg}(N, M)$. Applying Lemma II.2 inductively we conclude that $\operatorname{deg}(N, M)$ is finite, since we have assumed that the sets $\operatorname{deg}\left(N_{j, \mathbb{Q}}, M_{\mathbb{Q}}\right)$ and $\operatorname{deg}\left(M_{\mathbb{Q}}, M_{\mathbb{Q}}\right)$ are finite. But the monoid $\operatorname{Map}(N, N)$ of self-maps of $N$ acts by precomposition on the set $\operatorname{Map}(N, M)$ of maps from $N$ to $M$ and since $1 \in \operatorname{deg}(N, M)$ we see that there is an inclusion $\operatorname{deg}(N, N) \subset \operatorname{deg}(N, M)$. Hence $\operatorname{deg}(N, N)$ is finite and $N$ is inflexible.

In order to apply Theorem II.1 to our examples we shall need information about the group $\pi_{\operatorname{dim}} M-1(M) \otimes \mathbb{Q}$ for $M \in \mathcal{M}\left(A_{j},\left[A_{j}\right]\right)$ with $j=\{1, \ldots, 4\}$, where $A_{1}, \ldots, A_{4}$ are the dgas from Appendix I. Recall that $\pi_{*}(M) \otimes \mathbb{Q}$ is a $\mathbb{Q}$-vector space generated by the indecomposable elements of the minimal model of $M$ [7, Theorem 15.11]. Using the notation of Section I.1, it follows that

$$
\pi_{*}(M) \otimes \mathbb{Q} \cong \mathbb{Q}\left(x_{1}\right) \oplus \mathbb{Q}\left(x_{2}\right) \oplus \mathbb{Q}\left(y_{1}\right) \oplus \mathbb{Q}\left(y_{2}\right) \oplus \mathbb{Q}\left(y_{3}\right) \oplus \mathbb{Q}(z) .
$$

The degrees of the generators $x_{1}, x_{2}, y_{1}, y_{2}, y_{3}, z$ for each of the $A_{j}$ are listed in Section I.1. In particular we obtain: 
Lemma II.3 For all $j \in\{1, \ldots, 4\}$ and for all $M \in \mathcal{M}\left(A_{j},\left[A_{j}\right]\right)$, we have that $\pi_{n-1}(M) \otimes \mathbb{Q}=0$, where $n$ is the dimension of $M$.

Theorem II.1 allows us to prove the existence of large classes of inflexible manifolds. We do this systematically in Section II.3 and for now present the following simple example:

Example II.4 Let $j \in\{1, \ldots, 4\}$, let $M \in \mathcal{M}\left(A_{j},\left[A_{j}\right]\right)$, and let $r \in \mathbb{N}_{>0}$. Then the $r$-fold connected sum $M^{\# r}$ is inflexible (and simply connected). Looking at the rational cohomology ring of these manifolds shows $M^{\# r} \not M^{\# s}$, whenever $r \neq s$.

\section{II.2 Inflexible products}

In general, it is not clear that products of inflexible manifolds are inflexible as maps between products of manifolds cannot necessarily be decomposed into maps on the factors; we will show now that certain products of our basic examples of simply connected manifolds are inflexible:

Theorem II.5 (Inflexible products) Let $j \in\{2,3,4\}$, let $M \in \mathcal{M}\left(A_{j},\left[A_{j}\right]\right)$ be a manifold as in Theorem 6.8 and let $k \in \mathbb{N}_{>0}$. Then the $k$-fold product $M^{\times k}$ is inflexible (and simply connected).

This result is proved in the following by carefully analysing the algebraic counterpart, namely tensor products of the Poincare dgas $A_{2}, A_{3}$ and $A_{4}$ respectively. Recall that given dgas $A$ and $B$ there is the tensor product dga $A \otimes B$ [7, Example 3 on page 47] and that $H^{*}(A \otimes B) \cong H^{*}(A) \otimes H^{*}(B)$. In particular, if $(A,[A])$ and $(B,[B])$ are Poincaré dgas then so is the product $(A \otimes B,[A] \otimes[B])$.

Proposition II.6 For each $j \in\{2,3,4\}$ and for all $k \in \mathbb{N}_{>0}$ the $k$-fold tensor product $\left(A_{j}^{\otimes k},\left[A_{j}\right]^{\otimes k}\right)$ is an inflexible Poincaré dga.

Proof We shall give the proof for $A_{3}$ and then state the modifications necessary for $A_{2}$ and $A_{4}$. Let us fix some notation: for an index $a \in\{1, \ldots, k\}$ let $A_{3 a}$ denote the $a^{\text {th }}$ copy of $A_{3}$ in the $k$-fold product $A_{3}^{\otimes k}$. Similarly, for generators $x_{i}, y_{i} \in A_{3}$ as in Section I.1 let $x_{i a}$ and $y_{i a}$ denote the copy of $x_{i}$ or $y_{i}$ in $A_{3 a}$. Notice that because the fundamental class of each $A_{3 a}$ is given by $\left[x_{1 a}\right]^{26}$ the fundamental class of $A_{3}^{\otimes k}$ is given by $\otimes_{a=1}^{k}\left[x_{1 a}\right]^{26}$. Therefore, we can read off the degree of dga endomorphisms of $A_{3}^{\otimes k}$ by looking at the situation in degree $\left|x_{1}\right|=8$.

Now let $f: A_{3}^{\otimes k} \rightarrow A_{3}^{\otimes k}$ be a dga endomorphism of nonzero degree. Since $A_{3}^{\otimes k}$ is Poincaré with finite-dimensional cohomology it follows that $f$ induces isomorphisms 
on all cohomology groups, and so $f$ is a dga isomorphism [7, Proposition 12.10(i)]. Thus, $H^{8}(f): H^{8}\left(A_{3}^{\otimes k}\right) \rightarrow H^{8}\left(A_{3}^{\otimes k}\right)$ is a $\mathbb{Q}$-linear isomorphism. By construction of $A_{3}$, there is a canonical isomorphism $\left(A_{3}^{\otimes k}\right)^{8} \cong H^{8}\left(A_{3}^{\otimes k}\right)$ which identifies $H^{8}(f)$ with $\left.f\right|_{\left(A_{3}^{\otimes k}\right)^{8}}$. In particular, $\left.f\right|_{\left(A_{3}^{\otimes k}\right)^{k}}$ is also a $\mathbb{Q}$-linear isomorphism.

We shall show below that $H^{8}(f)$ is represented by a signed permutation matrix with respect to the obvious basis of $\left(A_{3}^{\otimes k}\right)^{8}=\left(A_{3}^{8}\right)^{\oplus k}$. If this holds, then, because $\bigotimes_{a=1}^{k}\left[x_{1 a}\right]^{26}$ is a fundamental class of $A_{3}^{\otimes k}$, the dga map $f$ has degree 1 or -1 , which proves that $A_{3}^{\times k}$ is inflexible.

In order to complete the proof it therefore remains to prove that $H^{8}(f)$ is represented by a signed permutation matrix. For each $b \in\{1, \ldots, k\}$ we have the dga projection $p_{b}: A_{3}^{\otimes k} \rightarrow A_{3 b}$ and the dga inclusion $i_{b}: A_{3 b} \rightarrow A_{3}^{\otimes k}$. Moreover, for $a, b \in\{1, \ldots, k\}$ we consider the dga map

$$
f_{a b}:=p_{a} \circ f \circ i_{b}: A_{3 b} \rightarrow A_{3 a} .
$$

Since $A_{3 a}=A_{3}=A_{3 b}$, we have by Proposition I.10 that $f_{a b}$ has degree 0,1 or -1 . Because $\left[A_{3}\right]=\left[x_{1}\right]^{26}$ and $A_{3}^{8}=\mathbb{Q} \cdot x_{1}$ it follows that $f_{a b}\left(x_{1 a}\right)= \pm x_{1 b}$ or $f_{a b}\left(x_{1 a}\right)=0$. Thus, for all $a \in\{1, \ldots, k\}$ we obtain

$$
f\left(x_{1 a}\right)=\sum_{b=1}^{k} \varepsilon_{a b} \cdot x_{1 b}, \quad \text { where } \varepsilon_{a b} \in\{-1,0,1\} .
$$

We proceed now by contradiction: suppose that for some $a$ at least two of the coefficients $\left\{\varepsilon_{a b} \mid b \in\{1, \ldots, k\}\right\}$ are nonzero. By construction of $A_{3}$ for $i \in\{10,33\}$ there are identifications $\left(A_{3}^{\otimes k}\right)^{i}=\bigoplus_{a=1}^{k} A_{3 a}^{i}$. We now consider the equation

$$
d f\left(y_{1 a}\right)=f\left(d y_{1 a}\right) .
$$

The left-hand side is a sum of monomials of the form $x_{1 c}^{3} x_{2 c}$, which can be seen by looking at the definition of $A_{3 a}^{33}$ and of the differential on $A_{3}$ (Example I.3). However, on the right-hand side, we have $f\left(d y_{1 a}\right)=f\left(x_{1 a}^{3} x_{2 a}\right)=f\left(x_{1 a}\right)^{3} \cdot f\left(x_{2 a}\right)$. Using the description of $f\left(x_{1 a}\right)$ from (3) and the fact that there are two nonzero coefficients $\varepsilon_{a b}$ and $\varepsilon_{a b^{\prime}}$, it follows that the right-hand side contains monomials of the form $\pm C_{b b^{\prime} c} \cdot x_{1 b}^{2} \cdot x_{1 b^{\prime}} \cdot x_{2 c}$, where $b \neq b^{\prime}$ and $C_{b b^{\prime} c} \in \mathbb{Q} \backslash\{0\}$. But such monomials are not present on the left-hand side, which is a contradiction. Therefore, we can conclude that for each $a \in\{1, \ldots, k\}$ only one of the coefficients $\varepsilon_{a b} \in\{-1,0,1\}$ is nonzero. As $H^{8}(f)=\left.f\right|_{\left(A_{3}^{\otimes k}\right)^{8}}$ is an isomorphism, it follows that $H^{8}(f)$ indeed is represented by a signed permutation matrix. 
For the dgas $A_{2}$ and $A_{4}$ the fundamental class is a power of $x_{2}$ and so we repeat the line of argument this time using $\left(A_{2}^{\otimes k}\right)^{10}=\bigoplus_{a=1}^{k} A_{2 a}^{10}$ or $\left(A_{4}^{\otimes k}\right)^{12}=\bigoplus_{a=1}^{k} A_{4 a}^{12}$ and the equation $d y_{2 a}=x_{1 a}^{2} x_{2 a}^{2}$ instead.

Notice that the above proof does not directly carry over to the case of the Poincare dga $\left(A_{1},\left[x_{2}\right]^{16}\right)$ because dga endomorphisms of $A_{1}$ are slightly more complicated in degree $\left|x_{2}\right|=4$ than in the cases discussed above.

Proof of Theorem II.5 The minimal model of $M^{\times k}$ is the $k$-fold tensor product $A_{j}^{\otimes k}$ [7, Example 1, page 248]; moreover, the fundamental class of $M^{k}$ corresponds to $\left[A_{j}\right]^{\otimes k} \in A_{j}^{\otimes k}$. Now the theorem follows because the Poincaré dga $A_{j}^{\otimes k}$ is inflexible by Proposition II.6.

Corollary II.7 In each of infinitely many dimensions there exist infinitely many rational homotopy types of oriented closed simply connected inflexible manifolds.

Proof Let $j \in\{2,3,4\}$, let $k \in \mathbb{N}_{>0}$, and let $r \in \mathbb{N}_{>0}$. Moreover, let $M \in$ $\mathcal{M}\left(A_{j},\left[A_{j}\right]\right)$. Theorems II.5 and II.1 (together with Lemma II.3) show that the oriented closed simply connected manifold $\left(M^{\times k}\right)^{\# r}$ is inflexible. The rational cohomology of these manifolds shows that if $r \neq r^{\prime}$, then $\left(M^{\times k}\right)^{\# r}$ and $\left(M^{\times k}\right)^{\# r^{\prime}}$ do not have the same rational homotopy type.

\section{II.3 Evidence for the genericity of inflexibility}

In the following, we combine results of the preceding sections to exhibit large numbers of simply connected inflexible manifolds. On the one hand, we show that there are "many" homotopy types of simply connected inflexible manifolds, and in particular that in many dimensions simply connected manifolds are "generic" from the point of view of oriented rational bordism. On the other hand, we show that simply connected inflexible manifolds exist that satisfy tangential structure constraints such as being parallelisable or nonspinable.

One way to create many (integral) homotopy types of simply connected inflexible manifolds out of a single inflexible Poincaré dga is to rescale the fundamental class of the dga in question:

Proposition II.8 (Scaling the fundamental class) Let $(A,[A])$ be an inflexible Poincaré dga, and let $a, a^{\prime} \in \mathbb{Q} \backslash\{0\}$ with $|a| \neq\left|a^{\prime}\right|$. If $M \in \mathcal{M}(A, a \cdot[A])$ and $M^{\prime} \in$ $\mathcal{M}\left(A, a^{\prime} \cdot[A]\right)$, then $M \not M^{\prime}$. 
Proof Recall that any Poincare dga is the minimal model of some simply connected rational $\mathbb{Q}$-Poincaré space (cf proof of Proposition 6.9); hence there is a rational $\mathbb{Q}$-Poincaré space $(X,[X])$ realising $(A,[A])$.

Let $M \in \mathcal{M}(A, a \cdot[A])$ and $M^{\prime} \in \mathcal{M}\left(A, a^{\prime} \cdot[A]\right)$; then the rationalisation of both $M$ and $M^{\prime}$ coincides with $X$, the only difference being that the fundamental classes are mapped to different multiples of $[X]$. Let $\rho_{M}: M \rightarrow M_{\mathbb{Q}}=X$ and $\rho_{M^{\prime}}: M^{\prime} \rightarrow X$ be the canonical maps provided by the rationalisation construction; by definition,

$$
H_{n}\left(\rho_{M} ; \mathbb{Q}\right)[M]_{\mathbb{Q}}=a \cdot[X] \text { and } H_{n}\left(\rho_{M^{\prime}} ; \mathbb{Q}\right)\left[M^{\prime}\right]_{\mathbb{Q}}=a^{\prime} \cdot[X],
$$

where $n:=\operatorname{dim} M=\operatorname{dim} M^{\prime}$. Assume for a contradiction that there is a homotopy equivalence $f: M \rightarrow M^{\prime}$. By the universal property of rationalisation [7, Theorem 9.7(ii)] there is a continuous map $f_{\mathbb{Q}}: X \rightarrow X$ with $\rho_{M^{\prime}} \circ f=f_{\mathbb{Q}} \circ \rho_{M}$. Hence

$$
\begin{aligned}
\operatorname{deg}_{[X]} f_{\mathbb{Q}} \cdot a \cdot[X] & =H_{n}\left(f_{\mathbb{Q}} \circ \rho_{M} ; \mathbb{Q}\right)[M]_{\mathbb{Q}} \\
& =H_{n}\left(\rho_{M^{\prime}} \circ f ; \mathbb{Q}\right)[M]_{\mathbb{Q}}=\operatorname{deg} f \cdot a^{\prime} \cdot[X] .
\end{aligned}
$$

Because $f$ is a homotopy equivalence and because $X$ is inflexible, it follows that $|\operatorname{deg} f|=1=\left|\operatorname{deg}_{[X]} f_{\mathbb{Q}}\right|$. Thus, $|a|=\left|a^{\prime}\right|$, a contradiction. So $M \not M^{\prime}$.

Example II.9 Let $j \in\{1, \ldots, 4\}$. In view of Remark 6.13, for all scalars $a \in \mathbb{Q} \backslash\{0\}$ the class $\mathcal{M}\left(A_{j}, a \cdot\left[A_{j}\right]\right)$ is nonempty. Therefore, by the proposition above, there are infinitely many homotopy types of oriented closed simply connected manifolds having the rational homotopy type given by $A_{j}$; clearly, all of these manifolds are inflexible.

Similarly, for $j \in\{2,3,4\}$ and all $k \in \mathbb{N}_{>0}$ there are infinitely many homotopy types of oriented closed simply connected manifolds having the rational homotopy type given by $A_{j}^{\otimes k}$ (because the corresponding Witt index is trivial as well, and so also the scalar multiples of the fundamental class are realisable by manifolds).

For Propositions II.10 and II.13 below we shall need the follow lemma, which is a refinement of a special case of the Barge-Sullivan Theorem 6.11:

Lemma II.10 Let $(X,[X])$ be a $\mathbb{Q}$-Poincaré space of formal dimension $4 k$ with vanishing Witt index $\tau_{[X]}=0 \in W_{0}(\mathbb{Q})$. Then $(X,[X])$ can be realised by a stably parallelisable oriented closed simply connected smooth manifold.

Proof The lemma follows from a little reflection upon the proof of the Barge-Sullivan theorem (Theorem 6.11). We need to find a stable bundle $\xi$ over the rational space $X$ such that the total Pontryagin class of $\xi$ is trivial; hence we may choose $\xi$ to be the 
trivial bundle. Since the manifold $M$ produced by the Barge-Sullivan theorem has a normal map

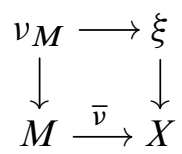

where $v_{M}$ is the stable normal bundle of $M, M$ is stably parallelisable.

Corollary II.11 For each of the example dgas $A_{1}, A_{2}, A_{3}$ and $A_{4}$ of Section I.1 and for each $a \in \mathbb{Q} \backslash\{0\}$, the class $\mathcal{M}\left(A_{j}, a \cdot\left[A_{j}\right]\right)$ contains a stably parallelisable manifold.

Proof By Proposition 6.12 the $\mathbb{Q}$-Poincaré spaces $\left(X_{j}, a \cdot\left[X_{j}\right]\right)$ realising the Poincaré dgas $\left(A_{j}, a \cdot\left[A_{j}\right]\right)$ all have vanishing Witt index, so we apply Lemma II.10.

In light of Theorem II.5 we introduce some notation: for $j \in\{1, \ldots, 4\}$ we write $d_{j}$ for the formal dimension of $A_{j}$; more explicitly, $d_{1}=64, d_{2}=108, d_{3}=208, d_{4}=228$. Moreover, we abbreviate

$$
\begin{aligned}
D & :=\left\{d_{1}\right\} \cup\left\{d_{j} \cdot k \mid k \in \mathbb{N}_{>0}, j \in\{2,3,4\}\right\} \\
& =\{64\} \cup\left\{d \cdot k \mid k \in \mathbb{N}_{>0}, d \in\{108,208,228\}\right\} .
\end{aligned}
$$

In dimensions in $D$ we will now show that simply connected inflexible manifolds are "generic" from the point of view of rational bordism, thereby giving a first answer in the direction of Question 6.16.

Proposition II.12 (Inflexible manifolds and rational bordism) Let $n \in D$. Then there is a positive integer $r(n)$, depending upon $n$, such that for any oriented closed $n-$ manifold $N$ the $r(n)$-fold disjoint union $\bigsqcup_{r(n)} N$, equivalently the $r$-fold connected sum $\#_{r(n)} N$, is oriented bordant to an oriented closed simply connected inflexible manifold.

Proof Because the products of complex projective spaces form a $\mathbb{Q}$-basis of the rational bordism ring $\Omega_{*}^{\mathrm{SO}} \otimes \mathbb{Q}\left[18\right.$, Corollary 18.9] and because the torsion in $\Omega_{*}^{\mathrm{SO}}$ has exponent 2 [25, Corollary 1] it suffices to consider the case where $N$ is a product of complex projective spaces, say $N=\prod_{i=1}^{m} \mathbb{C} P^{n_{i}}$ with $2 \cdot\left(n_{1}+\cdots+n_{m}\right)=n$.

By definition of $D$, we can write $n=d_{j} \cdot k$, with $j \in\{2,3,4\}$ and $k \in \mathbb{N}_{>0}$, or $j=1=k$. Moreover, let $M \in \mathcal{M}\left(A_{j},\left[A_{j}\right]\right)$; by Lemma II.10 we may assume that $M$ is stably parallelisable. Then $M^{\times k}$ is an oriented closed simply connected $n$-manifold that is inflexible (by Theorem II.5) and stably parallelisable. In particular, $M^{\times k}$ is oriented nullbordant. 
We now consider $N^{\prime}:=M^{\times k} \# N$. By construction, $N^{\prime}$ is oriented bordant to $N$ and simply connected. It hence suffices to show that $N^{\prime}$ is inflexible. By Lemma II.3, we have $\pi_{n-1}\left(M^{\times k}\right) \otimes \mathbb{Q} \cong \pi_{n-1}(M)^{\times k} \otimes \mathbb{Q}=0$. By definition, $H^{2}\left(M^{\times k} ; \mathbb{Q}\right)=0$ if $j>1$; in the case $n=d_{1}=64$, there is no class $x \in H^{2}(M ; \mathbb{Q})$ with $x^{32} \neq 0$ (by definition, $H^{2}(M ; \mathbb{Q}) \cong \mathbb{Q} \cdot x_{1}$, and $\left[x_{1}\right]^{32}=0$, as shown in the proof of Proposition I.5). However, there is a class $x \in H^{2}(N ; \mathbb{Q})=H^{2}\left(\prod_{i=1}^{m} \mathbb{C} P^{n_{i}}\right)$ such that $x^{n / 2}$ generates $H^{n}(N ; \mathbb{Q})$. Therefore, $\operatorname{deg}\left(N, M^{\times k}\right)=\{0\}$, and now applying Theorem II.1 shows that $N^{\prime}=M^{\times k} \# N$ is inflexible.

We saw above that there are many examples of stably parallelisable simply connected inflexible manifolds. On the other hand it is also possible to find simply connected inflexible manifolds with other tangential constraints. For example we have:

Proposition II.13 (Nonspinable inflexible manifolds) For all $n \in D$ there are oriented closed simply connected nonspinable inflexible manifolds of dimension $n$.

Proof Let $N=S^{n-2} \tilde{\times} S^{2}$ be the total space of the sphere bundle of the nontrivial rank $(n-1)$-vector bundle over $S^{2}$. Then the second Stiefel-Whitney class of $N$ generates $H^{2}(N ; \mathbb{Z} / 2)=\mathbb{Z} / 2$ and $N$ is nonspinable.

We write $n=d_{j} \cdot k$ with $j \in\{2,3,4\}$ and $k \in \mathbb{N}_{>0}$, or $j=1=k$. Then for all $M \in$ $\mathcal{M}\left(A_{j},\left[A_{j}\right]\right)$ the manifold $M^{\times k}$ is inflexible (by Theorem II.5) and simply connected. So $M^{\times k} \# N$ is nonspinable (because the Stiefel-Whitney class is nontrivial) and simply connected. We show now that $M^{\times k} \# N$ is inflexible.

As first step, we show that $\operatorname{deg}\left(N, M^{\times k}\right)=\{0\}$. A straightforward spectral sequence calculation shows that $H^{d}(N ; \mathbb{Q})=0$ for all $d \in\{4,6,8,12\}$. On the other hand, by construction of the Poincaré dgas $A_{1}, \ldots, A_{4}$ we have $H^{d}\left(M^{\times k} ; \mathbb{Q}\right) \neq 0$ for some $d \in\{4,6,8,12\}$. Therefore, $\operatorname{deg}\left(N, M^{\times k}\right)=\{0\}$.

Furthermore, from Lemma II.3 we obtain $\pi_{n-1}\left(M^{\times k}\right) \otimes \mathbb{Q}=0$. Hence, $M^{\times k} \# N$ is inflexible by Theorem II.1.

\section{References}

[1] M Amann, Mapping degrees of self-maps of simply-connected manifolds (2011) arXiv:1109.0960v1

[2] M Arkowitz, G Lupton, Rational obstruction theory and rational homotopy sets, Math. Z. 235 (2000) 525-539 MR1800210

[3] J Barge, Structures différentiables sur les types d'homotopie rationnelle simplement connexes, Ann. Sci. École Norm. Sup. 9 (1976) 469-501 MR0440574 
[4] J Barge, É Ghys, Surfaces et cohomologie bornée, Invent. Math. 92 (1988) 509-526 MR939473

[5] R Benedetti, C Petronio, Lectures on hyperbolic geometry, Springer, Berlin (1992) MR1219310

[6] C Costoya, A Viruel, Every finite group is the group of self-homotopy equivalences of an elliptic space, Acta Math. 213 (2014) 49-62 MR3261010

[7] Y Félix, S Halperin, J-C Thomas, Rational homotopy theory, Graduate Texts in Math. 205, Springer, New York (2001) MR1802847

[8] A A Gaifullin, Combinatorial realisation of cycles and small covers arXiv: 1204.0208

[9] A A Gaifullin, Universal realisators for homology classes, Geom. Topol. 17 (2013) 1745-1772 MR3073934

[10] M Gromov, Volume and bounded cohomology, Inst. Hautes Études Sci. Publ. Math. 56 (1982) 5-99 MR686042

[11] M Gromov, Metric structures for Riemannian and non-Riemannian spaces, Progress in Math. 152, Birkhäuser, Basel (1999) MR1699320

[12] N V Ivanov, Foundations of the theory of bounded cohomology, Zap. Nauchn. Sem. Leningrad. Otdel. Mat. Inst. Steklov. (LOMI) 143 (1985) 69-109, 177-178 MR806562 In Russian; translated in J. Soviet Math. 37 (1987) 1090-1114

[13] D Kotschick, C Löh, Fundamental classes not representable by products, J. Lond. Math. Soc. 79 (2009) 545-561 MR2506686

[14] M Kreck, Surgery and duality, Ann. of Math. 149 (1999) 707-754 MR1709301

[15] C Löh, Measure homology and singular homology are isometrically isomorphic, Math. Z. 253 (2006) 197-218 MR2206643

[16] C Löh, R Sauer, Degree theorems and Lipschitz simplicial volume for nonpositively curved manifolds of finite volume, J. Topol. 2 (2009) 193-225 MR2499443

[17] J W Milnor, D Husemoller, Symmetric bilinear forms, Ergeb. Math. Grenz. 73, Springer, Berlin (1973) MR0506372

[18] J W Milnor, J D Stasheff, Characteristic classes, Annals of Math. Studies 76, Princeton Univ. Press, Univ. Tokyo Press (1974) MR0440554

[19] J Neisendorfer, T Miller, Formal and coformal spaces, Illinois J. Math. 22 (1978) 565-580 MR0500938

[20] A Sambusetti, An obstruction to the existence of Einstein metrics on 4-manifolds, Math. Ann. 311 (1998) 533-547 MR1637923

[21] H Shiga, Rational homotopy type and self-maps, J. Math. Soc. Japan 31 (1979) 427-434 MR535089

[22] D Sullivan, Infinitesimal computations in topology, Inst. Hautes Études Sci. Publ. Math. 47 (1977) 269-331 MR0646078

[23] R Thom, Quelques propriétés globales des variétés différentiables, Comment. Math. Helv. 28 (1954) 17-86 MR0061823 
[24] W P Thurston, The geometry and topology of three-manifolds, lecture notes (1979) Available at http://msri.org/publications/books/gt3m/

[25] C T C Wall, Determination of the cobordism ring, Ann. of Math. 72 (1960) 292-311 MR0120654

Institute of Mathematics, University of Aberdeen

Aberdeen AB24 3UE, UK

Fakultät für Mathematik, Universität Regensburg

93040 Regensburg, Germany

dcrowley@abdn.ac.uk, clara.loeh@mathematik.uni-r.de

http://www.dcrowley.net, http://www.mathematik.uni-r.de/loeh

Received: 11 February 2014 
